\title{
A SOURCE TRANSFER DOMAIN DECOMPOSITION METHOD FOR HELMHOLTZ EQUATIONS IN UNBOUNDED DOMAIN
}

\author{
ZHIMING CHEN* AND XUESHUANG XIANG ${ }^{\dagger}$
}

\begin{abstract}
We propose and study a domain decomposition method for solving the truncated perfectly matched layer (PML) approximation in bounded domain of Helmholtz scattering problems. The method is based on the decomposition of the domain into non-overlapping layers and the idea of source transfer which transfers the sources equivalently layer by layer so that the solution in the final layer can be solved using a PML method defined locally outside the last two layers. The convergence of the method is proved for the case of constant wave number based on the analysis of the fundamental solution of the PML equation. The method can be used as an efficient preconditioner in the preconditioned GMRES method for solving discrete Helmholtz equations with constant and heterogeneous wave numbers. Numerical examples are included.
\end{abstract}

Key words. Helmholtz equation, high frequency waves, PML, source transfer.

1. Introduction. We propose and study a domain decomposition method for solving the Helmholtz scattering problem:

$$
\begin{aligned}
\Delta u+k^{2} u & =f \quad \text { in } \mathbb{R}^{2}, \\
r^{1 / 2}\left(\frac{\partial u}{\partial r}-\mathbf{i} k u\right) \rightarrow 0 & \text { as } r=|x| \rightarrow \infty,
\end{aligned}
$$

where $k>0$ is the wave number and $f \in H^{1}\left(\mathbb{R}^{2}\right)^{\prime}$ having compact support, where $H^{1}\left(\mathbb{R}^{2}\right)^{\prime}$ is the dual space of $H^{1}\left(\mathbb{R}^{2}\right)$. The results in this paper can be easily extended to solve three dimensional Helmholtz scattering problems. The proposed method can be used as an efficient preconditioner for discrete Helmholtz problems with constant and heterogeneous wave numbers $k(x)$.

Helmholtz equation (1.1) appears in diverse scientific and engineering applications including acoustics, elasticity, and electromagnetics. The efficient algebraic solver for large wave number discrete Helmholtz equation resulting from finite difference or finite element discretization is challenging due to the huge number of degrees of freedom required and the highly indefinite nature of the discrete problem. There exist considerable efforts in the literature for finding efficient algorithms for solving discrete Helmholtz equations, see e.g. Benamou and Després [4], Gander et al [16] for domain decomposition techniques and Brandt and Livshit [6], Elman et at [13] for multigrid methods. We refer to the review articles Erlangga [15], Osei-Kuffuor and Saad [22] and the references therein for further information. Our work is inspired by the recent work of Engquist and Ying [14] in which a sweeping preconditioner is constructed by an approximate $L D L^{t}$ factorization which eliminates the unknowns layer by layer. The Schur complement matrix of the factorization is approximated by using a moving perfectly matched layer (PML) technique.

In this paper we propose a new domain decomposition method for solving the Helmholtz problem (1.1)-(1.2). Let $\Omega_{i}=\left\{x=\left(x_{1}, x_{2}\right)^{T} \in \mathbb{R}^{2}: \zeta_{i}<x_{2}<\zeta_{i+1}\right\}$, $i=1, \cdots, N$, be the layers whose union covers the support of the source $f$. Let

*LSEC, Institute of Computational Mathematics, Academy of Mathematics and System Sciences, Chinese Academy of Sciences, Beijing 100190, China. This author was supported in part by National Basic Research Project under the grant 2011CB309700 and China NSF under the grant 11021101. (zmchen@lsec.cc.ac.cn).

${ }^{\dagger}$ LSEC, Institute of Computational Mathematics and Scientific/Engineering Computing, Academy of Mathematics and Systems Science, Chinese Academy of Sciences, Beijing 100190, China. (xiangxs@lsec.cc.ac.cn). 
$\Omega_{0}=\left\{x=\left(x_{1}, x_{2}\right)^{T} \in \mathbb{R}^{2}: x_{2}<\zeta_{1}\right\}$ and $\Omega_{N+1}=\left\{x=\left(x_{1}, x_{2}\right)^{T} \in \mathbb{R}^{2}: x_{2}>\zeta_{N+1}\right\}$. Let $f_{i}$ be the restriction of $f$ in $\Omega_{i}$ and vanish outside $\Omega_{i}$. It is clear that

$$
u(x)=-\int_{\mathbb{R}^{2}} f(y) G(x, y) d y=-\sum_{i=1}^{N} \int_{\Omega_{i}} f_{i}(y) G(x, y) d y, \quad G(x, y)=\frac{\mathbf{i}}{4} H_{0}^{(1)}(k|x-y|) .
$$

Let $\bar{f}_{1}=f_{1}$. The key idea in our method is that if one can find a proper way to transfer the source from $\Omega_{i}$ to $\Omega_{i+1}$ in the sense that

$$
\int_{\Omega_{i}} \bar{f}_{i}(y) G(x, y) d y=\int_{\Omega_{i+1}} \Psi_{i+1}\left(\bar{f}_{i}\right)(y) G(x, y) d y, \quad \forall x \in \Omega_{j}, \quad j>i+1,
$$

then for $\bar{f}_{i+1}=f_{i+1}+\Psi_{i+1}\left(\bar{f}_{i}\right)$ we have

$$
u(x)=-\int_{\Omega_{N}} f_{N}(y) G(x, y) d y-\int_{\Omega_{N-1}} \bar{f}_{N-1}(y) G(x, y) d y, \quad \forall x \in \Omega_{N} .
$$

We observe from (1.4) that the solution $u$ in $\Omega_{N}$ only involves the sources in $\Omega_{N}$ and $\Omega_{N-1}$ and thus can be solved locally by using the PML method defined outside only two layers $\Omega_{N}$ and $\Omega_{N-1}$. Once the solution $u$ in $\Omega_{N}$ is known, the solution in the other layers can be computed successively by solving the half-space Helmholtz problem using the transferred sources. The key step in the method is the source transfer algorithm resulting (1.3) and for this reason we call our algorithm the source transfer domain decomposition method (STDDM). We will make above heuristic idea rigorous in the setting of PML method.

The PML method is an efficient technique for solving wave scattering problems defined in unbounded domains. Since the work of Bérenger [5] which proposed a PML technique for solving the time dependent Maxwell equations, various constructions of PML absorbing layers have been proposed and studied in the literatures [23]. The basic idea of the PML technique is to surround the computational domain by a layer of finite thickness with specially designed model medium that would absorb all the waves that propagate from inside the computational domain. The convergence of the PML method for Helmholtz scattering problems is studied in Lassas and Sommersalo $[19,21]$ and Chen and Liu [9] for spherical and smooth PML layers, in Kim and Pasciak [18], Bramble and Pasciak [3], Chen and Wu [10], and Chen and Zheng [12] for uniaxial PML methods.

In section 2 we will introduce the STDDM in $\mathbb{R}^{2}$ which motivates the study of STDDM for truncated PML problem in section 3. We propose our method in the setting of uniaxial PML methods in which the complex coordinate stretching is performed along coordinate axises. The analysis in section 3 for the case of constant wave number shows the very desirable property of our STDDM method: for any fixed wave number, the computational complexity is proportional to the number of layers times the computational costs in each layer. In section 4 we use our method as a preconditioner for the GMRES method for solving discrete Helmholtz problems with both constant and heterogeneous wave number. The numerical experiments indicate that the number of preconditioned GMRES iterations is almost independent of the wave number if the discretization error is sufficiently controlled. We will report other possible extensions of STDDM including 3D problems, the problem with discontinuous wave numbers, and the scattering problem outside a bounded scatterer in a forthcoming paper [11]. 
2. The STDDM for the PML equation in $\mathbb{R}^{2}$. In this section we introduce the STDDM for the PML equation in the whole space. This provides the motivation for us to propose the STDDM for the PML equation in the truncated bounded domain in next section. We first recall some basic facts about the PML method in subsection 2.1 and then introduce the source transfer domain decomposition method in subsection 2.2 .

2.1. The PML method. Let the source $f$ be supported inside $B_{l}=\{x=$ $\left.\left(x_{1}, x_{2}\right)^{T} \in \mathbb{R}^{2}:\left|x_{1}\right|<l_{1},\left|x_{2}\right|<l_{2}\right\}$. It is known that the solution of the Helmholtz equation (1.1) with the Sommerfeld radiation condition (1.2) has the following integral representation

$$
u(x)=-\int_{\mathbb{R}^{2}} f(y) G(x, y) d y \text { in } \mathbb{R}^{2}, \quad G(x, y)=\frac{\mathbf{i}}{4} H_{0}^{(1)}(k|x-y|) .
$$

Here $H_{0}^{(1)}(z)$, for $z \in \mathbb{C}$, is the first Hankel function of order zero and $G(x, y)$ is the fundamental solution of the Helmholtz equation of constant wave number $k$

$$
\Delta G(x, y)+k^{2} G(x, y)=-\delta_{y}(x) \text { in } \mathbb{R}^{2} .
$$

The integral in (2.1) is well-defined if $f$ is a smooth function with compact support in $\mathbb{R}^{2}$. In the general case when $f \in H^{1}\left(\mathbb{R}^{2}\right)^{\prime}$ with compact support, it should be understood in the sense of distribution.

The PML method is based on the complex coordinate stretching outside the domain $B_{l}$ [8]. Let $\alpha_{1}\left(x_{1}\right)=1+\mathbf{i} \sigma_{1}\left(x_{1}\right), \alpha_{2}\left(x_{2}\right)=1+\mathbf{i} \sigma_{2}\left(x_{2}\right)$ be the model medium property. For $t \in \mathbb{R}, \sigma_{j}(t) \in C^{1}\left(\mathbb{R}^{2}\right)$ is a piecewise smooth function such that, for $j=1,2$,

$$
\sigma_{j}(t)=\sigma_{j}(-t), \quad \sigma_{j}=0 \text { for }|t| \leq l_{j}, \quad \text { and } \sigma_{j}=\gamma_{0}>0 \text { for }|t| \geq \bar{l}_{j},
$$

where $\bar{l}_{j}>l_{j}$ is fixed and $\gamma_{0}>0$ is a constant. This condition on the medium property is slightly different from that in $[18,3]$ but it still allows us to use the results in [18] on the fundamental solution of the PML equation and the uniqueness of the PML problem in $\mathbb{R}^{2}$. The key requirement here is $\sigma_{j}(t)$ is constant for $|t| \geq \bar{l}_{j}$ which is essential for using the reflection argument of [3] to prove the inf-sup condition for the PML problem in the truncated domain and to estimate the dependence of the inf-sup constants on the wave number $k$ when $k$ is large (see Lemma 3.4 below).

For $x=\left(x_{1}, x_{2}\right)^{T} \in \mathbb{R}^{2}$, denote by $\tilde{x}(x)=\left(\tilde{x}_{1}\left(x_{1}\right), \tilde{x}_{2}\left(x_{2}\right)\right)^{T}$ the complex coordinate, where

$$
\tilde{x}_{j}\left(x_{j}\right)=\int_{0}^{x_{j}} \alpha_{j}(t) d t=x_{j}+\mathbf{i} \int_{0}^{x_{j}} \sigma_{j}(t) d t, \quad j=1,2 .
$$

Notice that $\tilde{x}_{j}\left(x_{j}\right)$ depends only on $x_{j}$ and for this reason the method is called the uniaxial PML method. For any $z \in \mathbb{C}$, denote by $z^{1 / 2}$ the analytic branch of $\sqrt{z}$ such that $\operatorname{Re}\left(z^{1 / 2}\right)>0$ for any $z \in \mathbb{C} \backslash[0,+\infty)$. We define the complex distance

$$
\rho(\tilde{x}, \tilde{y})=\left[\left(\tilde{x}_{1}\left(x_{1}\right)-\tilde{y}_{1}\left(y_{1}\right)\right)^{2}+\left(\tilde{x}_{2}\left(x_{2}\right)-\tilde{y}_{2}\left(y_{2}\right)\right)^{2}\right]^{1 / 2} .
$$

The following lemma refines the result in [18, Lemma 3.3].

Lemma 2.1. Let $\sigma_{0}=\max _{x \in \mathbb{R}}\left(\sigma_{1}(t), \sigma_{2}(t)\right)>0$. Then we have

$$
\left(1+\max \left(1, \sigma_{0}\right)^{2}\right)^{-1 / 2}|x-y| \leq|\rho(\tilde{x}, \tilde{y})| \leq\left(1+\sigma_{0}^{2}\right)^{1 / 2}|x-y|, \quad \forall x, y \in \mathbb{R}^{2} .
$$


Proof. For the sake of completeness we recall the proof here. From the definition we know that

$$
\tilde{x}_{j}\left(x_{j}\right)-\tilde{y}_{j}\left(y_{j}\right)=\alpha_{j}\left(\xi_{j}\right)\left(x_{j}-y_{j}\right), \quad \alpha_{j}\left(\xi_{j}\right)=1+\mathbf{i} \sigma_{j}\left(\xi_{j}\right), \quad j=1,2,
$$

where $\xi_{j}$ is some number between $x_{j}$ and $y_{j}$. It is clear that $0 \leq \sigma_{j}\left(\xi_{j}\right) \leq \sigma_{0}$. Thus

$$
|\rho(\tilde{x}, \tilde{y})|^{2}=|x-y|^{2}\left|t_{1} \alpha_{1}\left(\xi_{1}\right)^{2}+t_{2} \alpha_{2}\left(\xi_{2}\right)^{2}\right|, \quad t_{j}=\left|x_{j}-y_{j}\right|^{2} /|x-y|^{2} .
$$

The right half of the desired estimate follows now directly since $t_{1}+t_{2}=1$. To obtain the lower bound of $|\rho(\tilde{x}, \tilde{y})|$ we first observe that

$$
\left|t_{1} \alpha_{1}\left(\xi_{1}\right)^{2}+t_{2} \alpha_{2}\left(\xi_{2}\right)^{2}\right|^{2}=t_{1}^{2}\left|\alpha_{1}\left(\xi_{1}\right)\right|^{4}+t_{2}^{2}\left|\alpha_{2}\left(\xi_{2}\right)\right|^{4}+2 t_{1} t_{2} \operatorname{Re}\left(\alpha_{1}\left(\xi_{1}\right)^{2} \overline{\alpha_{2}\left(\xi_{2}\right)^{2}}\right) .
$$

Write $\alpha_{j}\left(\xi_{j}\right)=\left|\alpha_{j}\left(\xi_{j}\right)\right| e^{\mathbf{i} \theta_{j}}$ with $0 \leq \theta_{j} \leq \operatorname{arctg}\left(\sigma_{0}\right)$ we deduce that

$$
\begin{aligned}
& \left|t_{1} \alpha_{1}\left(\xi_{1}\right)^{2}+t_{2} \alpha_{2}\left(\xi_{2}\right)^{2}\right|^{2} \\
= & t_{1}^{2}\left|\alpha_{1}\left(\xi_{1}\right)\right|^{4}+t_{2}^{2}\left|\alpha_{2}\left(\xi_{2}\right)\right|^{4}+2 t_{1} t_{2}\left|\alpha_{1}\left(\xi_{1}\right)\right|^{2}\left|\alpha_{2}\left(\xi_{2}\right)\right|^{2} \cos \left(2\left(\theta_{1}-\theta_{2}\right)\right) .
\end{aligned}
$$

If $2\left|\theta_{1}-\theta_{2}\right| \leq \pi / 2$, we have

$$
\left|t_{1} \alpha_{1}\left(\xi_{1}\right)^{2}+t_{2} \alpha_{2}\left(\xi_{2}\right)^{2}\right|^{2} \geq t_{1}^{2}\left|\alpha_{1}\left(\xi_{1}\right)\right|^{4}+t_{2}^{2}\left|\alpha_{2}\left(\xi_{2}\right)\right|^{4} \geq t_{1}^{2}+t_{2}^{2} \geq 1 / 2,
$$

where we have used the fact that $t_{j} \geq 0$ and $t_{1}+t_{2}=1$. Otherwise, if $2\left|\theta_{1}-\theta_{2}\right| \geq \pi / 2$, we have $2\left|\theta_{1}-\theta_{2}\right| \leq 2 \theta_{0}$, where $\pi / 4 \leq \theta_{0} \leq \pi / 2$ such that $\operatorname{tg} \theta_{0}=\sigma_{0}$. It is clear that in this case $\sigma_{0} \geq 1$ necessarily and $\cos 2 \theta_{0}=\left(1-\sigma_{0}^{2}\right) /\left(1+\sigma_{0}^{2}\right) \leq 0$. Thus

$$
\begin{aligned}
\left|t_{1} \alpha_{1}\left(\xi_{1}\right)^{2}+t_{2} \alpha_{2}\left(\xi_{2}\right)^{2}\right|^{2} & \geq t_{1}^{2}\left|\alpha_{1}\left(\xi_{1}\right)\right|^{4}+t_{2}^{2}\left|\alpha_{2}\left(\xi_{2}\right)\right|^{4}-2 t_{1} t_{2}\left|\alpha_{1}\left(\xi_{1}\right)\right|^{2}\left|\alpha_{2}\left(\xi_{2}\right)\right|^{2}\left|\cos 2 \theta_{0}\right| \\
& \geq\left(1-\left|\cos 2 \theta_{0}\right|\right)\left(t_{1}^{2}\left|\alpha_{1}\left(\xi_{1}\right)\right|^{4}+t_{2}^{2}\left|\alpha_{2}\left(\xi_{2}\right)\right|^{4}\right) \\
& \geq \frac{1}{2}\left(1-\left|\cos 2 \theta_{0}\right|\right)=\frac{1}{1+\sigma_{0}^{2}} .
\end{aligned}
$$

This completes the proof. $\square$

The following lemma proved in [9, Lemma 2.2] indicates that the first Hankel function decays exponentially away from the lower half complex plane.

Lemma 2.2. For any $\nu \in \mathbb{R}, z \in \mathbb{C}_{++}=\{z \in \mathbb{C}: \operatorname{Re}(z) \geq 0, \operatorname{Im}(z) \geq 0\}$, and $\Theta \in \mathbb{R}$ such that $0<\Theta \leq|z|$, we have

$$
\left|H_{\nu}^{(1)}(z)\right| \leq e^{-\operatorname{Im}(z)\left(1-\frac{\Theta^{2}}{|z|^{2}}\right)^{1 / 2}}\left|H_{\nu}^{(1)}(\Theta)\right| .
$$

The following estimate for the first Hankel function is proved in [7, (1.22)-(1.23)].

Lemma 2.3. For any $\Theta>0$, we have

$$
\left|H_{0}^{(1)}(\Theta)\right| \leq \sqrt{\frac{2}{\pi \Theta}}, \quad\left|H_{1}^{(1)}(\Theta)\right| \leq \sqrt{\frac{2}{\pi \Theta}}+\frac{2}{\pi \Theta} .
$$

The following elementary lemma is proved in [10, Lemma 3.1].

Lemma 2.4. For any $z_{1}=a_{1}+\mathbf{i} b_{1}, z_{2}=a_{2}+\mathbf{i} b_{2}$ with $a_{1}, a_{2}, b_{1}, b_{2} \in \mathbb{R}$ such that $a_{1} b_{1}+a_{2} b_{2} \geq 0$ and $a_{1}^{2}+a_{2}^{2}>0$, we have $\operatorname{Im}\left(z_{1}^{2}+z_{2}^{2}\right)^{1 / 2} \geq \frac{a_{1} b_{1}+a_{2} b_{2}}{\sqrt{a_{1}^{2}+a_{2}^{2}}}$.

By using Lemma 2.4 we know that

$$
\operatorname{Im} \rho(\tilde{x}, \tilde{y}) \geq \frac{1}{|x-y|}\left(\left|x_{1}-y_{1}\right|\left|\int_{y_{1}}^{x_{1}} \sigma_{1}(t) d t\right|+\left|x_{2}-y_{2}\right|\left|\int_{y_{2}}^{x_{2}} \sigma_{2}(t) d t\right|\right),
$$


which implies that $\operatorname{Im} \rho(\tilde{x}, \tilde{y}) \rightarrow \infty$ as $|x| \rightarrow \infty$ for any fixed $y \in \mathbb{R}$. The following lemma on the decay property of the stretched fundamental solution $G(\tilde{x}, \tilde{y})=$ $\frac{\mathbf{i}}{4} H_{0}^{(1)}(k \rho(\tilde{x}, \tilde{y}))$ is useful in our analysis.

LEMMA 2.5. There exists a positive constant $C$ independent of $k$ and the medium property $\sigma$ such that for any $x, y \in \mathbb{R}^{2}, x \neq y$,

$$
\begin{aligned}
& |G(\tilde{x}, \tilde{y})| \leq C\left(1+\sigma_{0}^{2}\right)^{1 / 4} e^{-\frac{1}{2} k \operatorname{Im} \rho(\tilde{x}, \tilde{y})}(k|x-y|)^{-1 / 2}, \\
& \left|\nabla_{x} G(\tilde{x}, \tilde{y})\right| \leq C k\left(1+\sigma_{0}^{2}\right)^{2} e^{-\frac{1}{2} k \operatorname{Im} \rho(\tilde{x}, \tilde{y})}\left[(k|x-y|)^{-1 / 2}+(k|x-y|)^{-1}\right], \\
& \left|\nabla_{x} \nabla_{y} G(\tilde{x}, \tilde{y})\right| \leq C k^{2}\left(1+\sigma_{0}^{2}\right)^{4} e^{-\frac{1}{2} k \operatorname{Im} \rho(\tilde{x}, \tilde{y})}\left[(k|x-y|)^{-1 / 2}+(k|x-y|)^{-2}\right] .
\end{aligned}
$$

Proof. By taking $\Theta=\frac{\sqrt{3}}{2} k|\rho(\tilde{x}, \tilde{y})|$ in Lemma 2.2 and using Lemma 2.3 we have

$$
\begin{aligned}
|G(\tilde{x}, \tilde{y})| & \leq \frac{1}{4} e^{-\frac{1}{2} k \operatorname{Im} \rho(\tilde{x}, \tilde{y})}\left|H_{0}^{(1)}\left(k \cdot \frac{\sqrt{3}}{2}|\rho(\tilde{x}, \tilde{y})|\right)\right| \\
& \leq C e^{-\frac{1}{2} k \operatorname{Im} \rho(\tilde{x}, \tilde{y})}|k \rho(\tilde{x}, \tilde{y})|^{-1 / 2} .
\end{aligned}
$$

This shows (2.6) by Lemma 2.1. The other two estimates (2.7) and (2.8) can be proved by a similar argument by using the identities

$$
\frac{d H_{0}^{(1)}(z)}{d z}=-H_{1}^{(1)}(z), \quad \frac{d H_{1}^{(1)}(z)}{d z}=H_{0}^{(1)}(z)-\frac{1}{z} H_{1}^{(1)}(z),
$$

and the fact that $\left|\nabla_{x} \rho(\tilde{x}, \tilde{y})\right| \leq|\alpha(x)||\tilde{x}-\tilde{y}| /|\rho(\tilde{x}, \tilde{y})| \leq\left(1+\max \left(1, \sigma_{0}\right)^{2}\right)^{3 / 2}$ by Lemma 2.1. $\mathrm{c}$

Now we define

$$
\tilde{u}(x)=u(\tilde{x})=-\int_{\mathbb{R}^{2}} f(y) G(\tilde{x}, \tilde{y}) d y, \quad \forall x \in \mathbb{R}^{2} .
$$

Since $f$ is supported inside $B_{l}$ we know that $\tilde{y}(y)=y$ and $\tilde{u}$ is well-defined in $H_{\text {loc }}^{1}\left(\mathbb{R}^{2}\right)$ and decays exponentially as $|x| \rightarrow \infty$. Obviously $\tilde{u}=u$ in $B_{l}$ and $\tilde{u}$ satisfies $\tilde{\Delta} \tilde{u}+$ $k^{2} \tilde{u}=f$ in $\mathbb{R}^{2}$, where $\tilde{\Delta}$ is the Laplacian with respect to the stretched coordinate $\tilde{x}$. This yields by the chain rule that $\tilde{u}$ satisfies the PML equation

$$
J^{-1} \nabla \cdot(A \nabla \tilde{u})+k^{2} \tilde{u}=f \quad \text { in } \mathbb{R}^{2},
$$

where $A(x)=\operatorname{diag}\left(\frac{\alpha_{2}\left(x_{2}\right)}{\alpha_{1}\left(x_{1}\right)}, \frac{\alpha_{1}\left(x_{1}\right)}{\alpha_{2}\left(x_{2}\right)}\right)$ is a diagonal matrix and $J(x)=\alpha_{1}\left(x_{1}\right) \alpha_{2}\left(x_{2}\right)$.

The fundamental solution of the PML equation (2.10) is (cf. e.g. [21, Theorem $2.8]$ and [3, Theorem 4.1])

$$
\tilde{G}(x, y)=J(y) G(\tilde{x}, \tilde{y})=\frac{\mathbf{i}}{4} J(y) H_{0}^{(1)}(k \rho(\tilde{x}, \tilde{y})),
$$

which satisfies

$$
J^{-1} \nabla \cdot(A \nabla \tilde{G}(x, y))+k^{2} \tilde{G}(x, y)=-\delta_{y}(x) \text { in } \mathbb{R}^{2} .
$$

Notice that $\tilde{G}(x, y) \neq \tilde{G}(y, x)$. It is known that $\tilde{G}(y, x)$ is the fundamental solution of the adjoint equation [21, Theorem 2.8]

$$
\nabla \cdot\left(A \nabla\left(J^{-1} \tilde{G}(y, x)\right)\right)+k^{2} \tilde{G}(y, x)=-\delta_{y}(x) \text { in } \mathbb{R}^{2} .
$$

Here we recall that $\nabla \cdot A \nabla J^{-1}$ is the adjoint operator of $J^{-1} \nabla \cdot A \nabla$. 
2.2. STDDM for the PML equation in $\mathbb{R}^{2}$. We start by introducing some notation. For any $a, b \in \mathbb{R}$, we denote $\Omega(a, b)=\left\{x=\left(x_{1}, x_{2}\right)^{T} \in \mathbb{R}^{2}: a<x_{2}<b\right\}$. We also use the notation $\Omega(-\infty, b)$ for the half-space $\left\{x=\left(x_{1}, x_{2}\right)^{T} \in \mathbb{R}^{2}: x_{2}<b\right\}$. Let $-l_{2}=\zeta_{1}<\zeta_{2}<\cdots<\zeta_{N+1}=l_{2}, N>1$, be a division of the interval $\left(-l_{2}, l_{2}\right)$. For simplicity, we will assume in this paper $\zeta_{i}=\zeta_{1}+(i-1) \Delta \zeta$, where $\Delta \zeta=2 l_{2} / N$. The general case of non-equally spaced division can be considered similarly. Let $\Omega_{i}=\Omega\left(\zeta_{i}, \zeta_{i+1}\right)$ and $\Gamma_{i}=\left\{x=\left(x_{1}, x_{2}\right)^{T} \in \mathbb{R}^{2}: x_{2}=\zeta_{i}\right\}$.

We define $f_{i}=\left.f\right|_{\Omega_{i}}$ in $\Omega_{i}$ and $f_{i}=0$ in $\mathbb{R}^{2} \backslash \bar{\Omega}_{i}$. For $x=\left(x_{1}, x_{2}\right)^{T} \in \Omega_{i}$, we denote by $\beta_{i}=\beta_{i}\left(x_{2}\right)$ a smooth function such that

$$
\beta_{i}=1, \beta_{i}^{\prime}=0 \text { on } \Gamma_{i}, \quad \beta_{i}=\beta_{i}^{\prime}=0 \text { on } \Gamma_{i+1}, \quad\left|\beta_{i}^{\prime}\right| \leq C(\Delta \zeta)^{-1} \text { in }\left(\zeta_{i}, \zeta_{i+1}\right) .
$$

We remark that this assumption will be assumed throughout the paper. The bound $\left|\beta_{i}^{\prime}\right| \leq C(\Delta \zeta)^{-1}$ will be used in the proof Lemma 3.6.

Our source transfer domain decomposition method consists of two steps. The first step is a source transfer algorithm we define now.

\section{Algorithm 2.1. (Source transfer for PML PROBlem In $\mathbb{R}^{2}$ )}

$1^{\circ}$ Let $\bar{f}_{1}=f_{1}$ in $\mathbb{R}^{2}$;

$2^{\circ}$ While $i=1,2, \cdots, N-2$,

- Find $u_{i} \in H^{1}\left(\mathbb{R}^{2}\right)$ such that

$$
J^{-1} \nabla \cdot\left(A \nabla u_{i}\right)+k^{2} u_{i}=-\bar{f}_{i} \text { in } \mathbb{R}^{2} .
$$

- Compute

$$
\Psi_{i+1}\left(\bar{f}_{i}\right)=J^{-1} \nabla \cdot\left(A \nabla\left(\beta_{i+1} u_{i}\right)\right)+k^{2}\left(\beta_{i+1} u_{i}\right) \quad \text { in } \Omega_{i+1} .
$$

- Set $\bar{f}_{i+1}=f_{i+1}+\Psi_{i+1}\left(\bar{f}_{i}\right)$ in $\Omega_{i+1}$ and $\bar{f}_{i+1}=0$ elsewhere.

End while

By (2.12) we know that $u_{i}$ is given by

$$
u_{i}(x)=\int_{\Omega_{i}} \bar{f}_{i}(y) J(y) G(\tilde{x}, \tilde{y}) d y, \quad \forall x \in \mathbb{R}^{2} .
$$

The following lemma whose proof will be given in the Appendix plays a key role in the analysis of this paper.

Lemma 2.6. For $i=1, \cdots, N-2$, we have $u_{i} \in H^{1}\left(\mathbb{R}^{2}\right)$ and $\left\|u_{i}\right\|_{H^{1}\left(\mathbb{R}^{2}\right)} \leq$ $C\|f\|_{H^{1}\left(B_{l}\right)^{\prime}}$. Let $M_{0}=l, M_{i}=\sqrt{2} M+(1+\sqrt{2}) M_{i-1}$, where $l$ is the diameter of $B_{l}$ and $M=\max \left(\bar{l}_{1}, \bar{l}_{2}\right)$. Then there exists a constant $C>0$ such that

$$
\left|u_{i}(x)\right|+\left|\nabla u_{i}(x)\right| \leq C e^{-\frac{1}{8} k \gamma_{0}|x|}\|f\|_{H^{1}\left(B_{l}\right)^{\prime}}, \quad \forall|x| \geq M_{i},
$$

where $H^{1}\left(B_{l}\right)^{\prime}$ is the dual space of $H^{1}\left(B_{l}\right)$.

We remark that the constants $C$ in Lemma 2.6 may depend on $k, \sigma_{0}, \gamma_{0}$. In the following we will only use the consequence of this lemma that $\left|u_{i}(x)\right|$ and $\left|\nabla u_{i}(x)\right|$ decay exponentially as $|x| \rightarrow \infty$. How the constants $C$ depend on $k, \sigma_{0}, \gamma_{0}$ is not important for the analysis of the STDDM in this paper.

Since $\bar{f}_{i}=0$ in $\Omega_{i+1}$, we know that

$$
\Psi_{i+1}\left(\bar{f}_{i}\right)=J^{-1} \frac{\partial}{\partial x_{2}}\left(\frac{\alpha_{1}}{\alpha_{2}} \beta_{i+1}^{\prime} u_{i}\right)+J^{-1} \beta_{i+1}^{\prime} \frac{\alpha_{1}}{\alpha_{2}} \frac{\partial u_{i}}{\partial x_{2}} \text { in } \Omega_{i+1} .
$$


By Lemma 2.6 and (2.17) we know that $\Psi_{i+1}\left(\bar{f}_{i}\right) \in L^{2}\left(\Omega_{i+1}\right)$. The following theorem is the main result of this section.

THEOREM 2.7. The following assertions hold:

(i) For $i=1, \cdots, N-2$, we have, for any $x \in \Omega\left(\zeta_{N},+\infty\right)$,

$$
\int_{\Omega_{i}} \bar{f}_{i}(y) \tilde{G}(x, y) d y=\int_{\Omega_{i+1}} \Psi_{i+1}\left(\bar{f}_{i}\right)(y) \tilde{G}(x, y) d y .
$$

(ii) The stretched PML function $\tilde{u}$ in (2.9) satisfies $\tilde{u}=v_{N}$ in $\Omega\left(\zeta_{N},+\infty\right)$, where

$$
v_{N}(x)=-\int_{\Omega_{N}} f_{N}(y) \tilde{G}(x, y) d y-\int_{\Omega_{N-1}} \bar{f}_{N-1}(y) \tilde{G}(x, y) d y \text { in } \mathbb{R}^{2} .
$$

Proof. Since $\tilde{y}(y)=y$ and $J(y)=1$ for $y \in B_{l}$, we know that

$$
\tilde{u}(x)=-\sum_{i=1}^{N} \int_{\Omega_{i}} f_{i}(y) G(\tilde{x}, \tilde{y}) d y=-\sum_{i=1}^{N} \int_{\Omega_{i}} f_{i}(y) \tilde{G}(x, y) d y .
$$

This shows (ii) if (i) is proved. To show (i) we first note that by (2.13)

$\nabla_{y} \cdot\left(A \nabla_{y}\left(J^{-1} \tilde{G}(x, y)\right)\right)+k^{2} J\left(J^{-1} \tilde{G}(x, y)\right)=0, \quad \forall x \in \Omega\left(\zeta_{N},+\infty\right), \forall y \in \Omega\left(\zeta_{1}, \zeta_{N}\right)$.

Now for $x \in \Omega\left(\zeta_{N},+\infty\right), y \in \Omega_{i}, i=1,2, \cdots, N-1, \tilde{G}(x, y)$ is a smooth function which decays exponentially as $|y| \rightarrow \infty$. By Lemma 2.6 we know that $u_{i}(y)$ decays also exponentially at infinity, we can do integration by parts to have

$$
\begin{aligned}
& \int_{\Omega_{i}} \bar{f}_{i}(y) \tilde{G}(x, y) d y \\
= & -\int_{\Omega\left(-\infty, \zeta_{i+1}\right)} J^{-1}\left[\nabla_{y} \cdot\left(A \nabla_{y} u_{i}(y)\right)+k^{2} J u_{i}(y)\right] \tilde{G}(x, y) d y \\
= & -\int_{\Gamma_{i+1}}\left[\left(A \nabla_{y} u_{i}(y) \cdot e_{2}\right) J^{-1} \tilde{G}(x, y)-\left(A \nabla_{y}\left(J^{-1} \tilde{G}(x, y)\right) \cdot e_{2}\right) u_{i}(y)\right] d s(y),
\end{aligned}
$$

where $e_{2}$ is the unit vector in the $x_{2}$ axis. By (2.14) and integrating by parts we have then

$$
\begin{aligned}
& \int_{\Omega_{i}} \bar{f}_{i}(y) \tilde{G}(x, y) d y \\
= & \int_{\partial \Omega_{i+1}}\left[\left(A \nabla_{y}\left(\beta_{i+1} u_{i}(y)\right) \cdot n\right)\left(J^{-1} \tilde{G}(x, y)\right)-\left(A \nabla_{y}\left(J^{-1} \tilde{G}(x, y)\right) \cdot n\right)\left(\beta_{i+1} u_{i}(y)\right)\right] d s(y) \\
= & \int_{\Omega_{i+1}}\left[\nabla_{y} \cdot\left(A \nabla_{y}\left(\beta_{i+1} u_{i}(y)\right)\right)+k^{2} J\left(\beta_{i+1} u_{i}(y)\right)\right] J^{-1} \tilde{G}(x, y) d y \\
= & \int_{\Omega_{i+1}} \Psi_{i+1}\left(\bar{f}_{i}\right)(y) \tilde{G}(x, y) d y
\end{aligned}
$$

where $n$ is the unit outer normal to $\partial \Omega_{i+1}$. This completes the proof. $\square$

The identity (2.18) means that for the solution in $\Omega_{N}$, the source $\bar{f}_{i}$ in $\Omega_{i}$ is equivalently transferred to the source $\Psi_{i+1}\left(\bar{f}_{i}\right)$ in $\Omega_{i+1}, i=1, \cdots, N-2$. It is easy to see that $v_{N}$ in Theorem 2.7 satisfies the equation

$$
J^{-1} \nabla \cdot\left(A \nabla v_{N}\right)+k^{2} v_{N}=f_{N}+\bar{f}_{N-1} \text { in } \mathbb{R}^{2} .
$$


Once $v_{N}$ is known, the solution $\tilde{u}$ in $\Omega_{i}, i=N-1, \cdots, 1$, can be solved successively by the following wave expansion algorithm.

Algorithm 2.2. (Wave expansion for PML Problem IN $\mathbb{R}^{2}$ ) $1^{\circ}$ Solve $v_{N} \in H^{1}\left(\mathbb{R}^{2}\right)$ such that

$$
J^{-1} \nabla \cdot\left(A \nabla v_{N}\right)+k^{2} v_{N}=f_{N}+\bar{f}_{N-1} \quad \text { in } \mathbb{R}^{2} .
$$

$2^{\circ}$ For $i=N-1, \cdots, 2$, find $v_{i} \in H^{1}\left(\Omega\left(-\infty, \zeta_{i+1}\right)\right)$ such that

$$
\begin{aligned}
J^{-1} \nabla \cdot\left(A \nabla v_{i}\right)+k^{2} v_{i} & =f_{i}+\bar{f}_{i-1} \quad \text { in } \Omega\left(-\infty, \zeta_{i+1}\right), \\
v_{i} & =v_{i+1} \quad \text { on } \Gamma_{i+1} .
\end{aligned}
$$

The second step of Algorithm 2.2 is to solve the PML problem in the half-space $\Omega\left(-\infty, \zeta_{i+1}\right)$ with Dirichlet boundary condition on $\Gamma_{i+1}$. The uniqueness and existence of the half-space PML problem can be proved by extending the method in [18]. In this paper we will not dwell on the details. The following theorem indicates that $v_{i}$ is indeed the solution $\tilde{u}$ of $(2.9)$ in $\Omega_{i}$.

TheOREM 2.8. We have $\tilde{u}=v_{N}$ in $\Omega\left(\zeta_{N},+\infty\right), \tilde{u}=v_{i}$ in $\Omega_{i}$ for all $i=$ $N-1, \cdots, 3$, and $\tilde{u}=v_{2}$ in $\Omega\left(-\infty, \zeta_{3}\right)$.

Proof. The case of $i=N$ is proved in the second part of Theorem 2.7. To prove the case of $i=N-1, \cdots, 3$, we first use the image method to construct the Green function $\Phi_{i+1}(x, y)$ for the half-space PML problem

$$
\begin{aligned}
J^{-1} \nabla \cdot\left(A \nabla \Phi_{i+1}(x, y)\right)+k^{2} \Phi_{i+1}(x, y) & =-\delta_{y}(x) \quad \text { in } \Omega\left(-\infty, \zeta_{i+1}\right), \\
\Phi_{i+1} & =0 \quad \text { on } \Gamma_{i+1} .
\end{aligned}
$$

For $x=\left(x_{1}, x_{2}\right)^{T} \in \mathbb{R}^{2}$, let $\tilde{x}_{i+1}^{R}(x)=\left(\tilde{x}_{i+1,1}^{R}\left(x_{1}\right), \tilde{x}_{i+1,2}^{R}\left(x_{2}\right)\right)^{T}$ be the complex coordinate stretching outside the domain $\left(-l_{1}, l_{1}\right) \times\left(\zeta_{1}, 2 \zeta_{i+1}-\zeta_{1}\right)$, where $\tilde{x}_{i+1,1}^{R}\left(x_{1}\right)=$ $\tilde{x}_{1}\left(x_{1}\right)$ and

$$
\tilde{x}_{i+1,2}^{R}\left(x_{2}\right)= \begin{cases}x_{2}+\mathbf{i} \int_{\zeta_{1}}^{x_{2}} \sigma_{2}(t) d t & \text { if } x_{2}<\zeta_{1} \\ x_{2} & \text { if } \zeta_{1} \leq x_{2} \leq 2 \zeta_{i+1}-\zeta_{1}, \\ x_{2}+\mathbf{i} \int_{2 \zeta_{i+1}-\zeta_{1}}^{x_{2}} \sigma_{2}\left(2 \zeta_{i+1}-t\right) d t & \text { if } x_{2}>2 \zeta_{i+1}-\zeta_{1}\end{cases}
$$

Let $A_{i+1}^{R}(x)=\operatorname{diag}\left(\frac{\tilde{x}_{i+1,2}^{R}\left(x_{2}\right)^{\prime}}{\tilde{x}_{i+1,1}^{R}\left(x_{1}\right)^{\prime}} \frac{\tilde{x}_{i+1,1}^{R}\left(x_{1}\right)^{\prime}}{\tilde{x}_{i+1,2}^{R}\left(x_{2}\right)^{\prime}}\right)$ and $J_{i+1}^{R}(x)=\tilde{x}_{i+1,1}^{R}\left(x_{1}\right)^{\prime} \tilde{x}_{i+1,2}^{R}\left(x_{2}\right)^{\prime}$. Obviously $A_{i+1}^{R}(x)=A(x)$ and $J_{i+1}^{R}(x)=J(x)$ for $x \in \Omega\left(-\infty, \zeta_{i+1}\right)$. It is clear that $\tilde{G}_{i+1}^{R}(x, y)=J_{i+1}^{R}(y) G\left(\tilde{x}_{i+1}^{R}(x), \tilde{y}_{i+1}^{R}(y)\right)$ is the fundamental solution of the PML equation

$$
\left(J_{i+1}^{R}\right)^{-1} \nabla \cdot\left(A_{i+1}^{R} \nabla \tilde{G}_{i+1}^{R}(x, y)\right)+k^{2} \tilde{G}_{i+1}^{R}(x, y)=-\delta_{y}(x) \quad \text { in } \mathbb{R}^{2} .
$$

The Green function of the half-space PML problem is $\Phi_{i+1}(x, y)=\tilde{G}_{i+1}^{R}(x, y)-$ $\tilde{G}_{i+1}^{R}\left(x, R_{i+1}(y)\right)$, where $R_{i+1}(y)=\left(y_{1}, 2 \zeta_{i+1}-y_{2}\right)^{T}$ is the image point of $y$ with respect to $\Gamma_{i+1}$. By $(2.13)$ and the fact that $J_{i+1}^{R}\left(R_{i+1}(y)\right)=J_{i+1}^{R}(y)$ for $y \in$ $\Omega\left(-\infty, \zeta_{i+1}\right)$ we have

$$
\nabla_{y} \cdot\left(A(y) \nabla_{y}\left(J^{-1} \Phi_{i+1}(x, y)\right)\right)+k^{2} \Phi_{i+1}(x, y)=0 \text { for } x \in \Omega_{i+1}, y \in \Omega\left(\zeta_{1}, \zeta_{i}\right),
$$


which implies, by the argument in the proof of Theorem 2.7 (i), that

$$
\int_{\Omega_{j}} \bar{f}_{j}(y) \Phi_{i+1}(x, y) d y=\int_{\Omega_{j+1}} \Psi_{j+1}\left(\bar{f}_{j}\right)(y) \Phi_{i+1}(x, y) d y \text { in } \Omega_{i},
$$

for any $j=1, \cdots, i-2$.

Now we prove the case for $i=N-1, \cdots, 3$. Since $\tilde{u}=v_{i+1}$ in $\Omega_{i+1}$ and thus on $\Gamma_{i+1}$, we know from (2.10) and (2.20)-(2.21) that $w_{i}=\tilde{u}-v_{i} \in H^{1}\left(\Omega\left(-\infty, \zeta_{i+1}\right)\right)$ satisfies

$$
\begin{aligned}
J^{-1} \nabla \cdot\left(A \nabla w_{i}\right)+k^{2} w_{i} & =f-\left(f_{i}+\bar{f}_{i-1}\right) \quad \text { in } \Omega\left(-\infty, \zeta_{i+1}\right), \\
w_{i} & =0 \quad \text { on } \Gamma_{i+1} .
\end{aligned}
$$

Since $f=f_{i}$ in $\Omega_{i}$ and $f$ vanishes in $\Omega\left(-\infty, \zeta_{1}\right)$ we know that

$$
\begin{aligned}
w_{i}(x) & =-\int_{\Omega\left(\zeta_{1}, \zeta_{i}\right)} f(y) \Phi_{i+1}(x, y) d y+\int_{\Omega_{i-1}} \bar{f}_{i-1}(y) \Phi_{i+1}(x, y) d y \\
& =-\sum_{j=1}^{i-2} \int_{\Omega_{j}} f_{j}(y) \Phi_{i+1}(x, y) d y+\int_{\Omega_{i-1}} \Psi_{i-1}\left(\bar{f}_{i-2}\right)(y) \Phi_{i+1}(x, y) d y .
\end{aligned}
$$

By repeatedly using (2.22) we obtain $w_{i}=0$ in $\Omega_{i}$.

The final case when $i=2$ is simple. Since $\bar{f}_{1}=f_{1}$ by definition, we know that $w_{2}=\tilde{u}-v_{2}$ satisfies (2.23)-(2.24) with vanishing source. This implies $\tilde{u}=v_{2}$ in $\Omega\left(-\infty, \zeta_{3}\right)$ and thus completes the proof.

The following integral representation for the solution $v_{i}$ of (2.20)-(2.21) can be proved by standard argument

$$
\begin{aligned}
v_{i}(x)= & -\int_{\Omega\left(\zeta_{i-1}, \zeta_{i+1}\right)}\left(f_{i}+\bar{f}_{i-1}\right)(y) \Phi_{i+1}(x, y) d y \\
& -\int_{\Gamma_{i+1}} \frac{\partial \Phi_{i+1}(x, y)}{\partial y_{2}} v_{i+1}(y) d s(y) \quad \text { in } \Omega\left(-\infty, \zeta_{i+1}\right) .
\end{aligned}
$$

Theorem 2.8 indicates that we can solve the PML problem (2.10) by solving $N-2$ half-space PML problem (2.20)-(2.21), $N-2$ full space PML problem (2.15) in the source transfer algorithm, and one full space PML problem (2.19). They are the starting point of the STDDM algorithm for the truncated PML problem to be introduced in the next section.

3. The STDDM for PML equation in the truncated bounded domain. In this section we study the STDDM method for the truncated PML equation. We start by introducing some notation to be used throughout this paper. For any bounded domain $U \subset \mathbb{R}^{2}$ with Lipschitz boundary $\Gamma$, we will use the weighted $H^{1}(U)$ norm $\|u\|_{H^{1}(U)}=\left(\|\nabla u\|_{L^{2}(U)}^{2}+\|k u\|_{L^{2}(U)}^{2}\right)^{1 / 2}$ and the weighted $H^{1 / 2}(\Gamma)$ norm $\|v\|_{H^{1 / 2}(\Gamma)}=\left(d_{U}^{-1}\|v\|_{L^{2}(\Gamma)}^{2}+|v|_{\frac{1}{2}, \Gamma}^{2}\right)^{1 / 2}$, where $d_{U}$ is the diameter of $U$, and

$$
|v|_{\frac{1}{2}, \Gamma}^{2}=\int_{\Gamma} \int_{\Gamma} \frac{\left|v(x)-v\left(x^{\prime}\right)\right|^{2}}{\left|x-x^{\prime}\right|^{2}} d s(x) d s\left(x^{\prime}\right) .
$$

It is obvious that

$$
\|v\|_{H^{1 / 2}(\Gamma)} \leq\left(|\Gamma| d_{U}^{-1}\right)^{1 / 2}\|v\|_{L^{\infty}(\Gamma)}+|\Gamma|\|\nabla v\|_{L^{\infty}(\Gamma)}, \quad \forall v \in W^{1, \infty}(\Gamma) .
$$


By the scaling argument and trace theorem we know that there exist constants $C_{1}, C_{2}>0$ independent of $d_{U}$ such that for any $v \in H^{1 / 2}(\Gamma)$,

$$
C_{1} \frac{|U|^{\frac{1}{2}}}{|\Gamma|}\|v\|_{H^{1 / 2}(\Gamma)} \leq \inf _{\left.\phi\right|_{\Gamma}=v, \phi \in H^{1}(U)}\|\phi\|_{H^{1}(U)} \leq C_{2} \frac{|U|^{\frac{1}{2}}}{d_{U}}\|v\|_{H^{1 / 2}(\Gamma)},
$$

where $\|\phi\|_{H^{1}(U)}=\left(\|\nabla \phi\|_{L^{2}(U)}^{2}+d_{U}^{-2}\|\phi\|_{L^{2}(U)}^{2}\right)^{1 / 2}$.

Let $B_{L}=\left(-l_{1}-d_{1}, l_{1}+d_{1}\right) \times\left(-l_{2}-d_{2}, l_{2}+d_{2}\right), l_{1}+d_{1}>\bar{l}_{1}, l_{2}+d_{2}>\bar{l}_{2}$, be the rectangle that includes $B_{l}$. The PML problem whose solution approximates $\tilde{u}$ in (2.10) in the bounded domain $B_{L}$ is to find $\hat{u} \in H_{0}^{1}\left(B_{L}\right)$ such that

$$
(A \nabla \hat{u}, \nabla \psi)-k^{2}(J \hat{u}, \psi)=-\langle J f, \psi\rangle, \quad \forall \psi \in H_{0}^{1}\left(B_{L}\right),
$$

where $(\cdot, \cdot)$ is the inner product in $L^{2}\left(\mathbb{R}^{2}\right)$ and $\langle\cdot, \cdot\rangle$ is the duality pairing between $H^{1}\left(\mathbb{R}^{2}\right)^{\prime}$ and $H^{1}\left(\mathbb{R}^{2}\right)$. We remark that since $\psi \in H_{0}^{1}\left(B_{L}\right)$, the inner product and the duality pairing in (3.4) are in fact taken in the domain $B_{L}$.

Throughout this section we assume that the medium property satisfies

(H1) $l_{1} \leq l_{2}, d_{1}=2 d_{2}$, and

$$
\int_{l_{1}}^{l_{1}+d_{2}} \sigma_{1}(t) d t=\int_{l_{2}}^{l_{2}+d_{2}} \sigma_{2}(t) d t=: \bar{\sigma}, \quad \int_{l_{1}+d_{2}}^{l_{1}+d_{1}} \sigma_{1}(t) d t \geq \bar{\sigma} .
$$

This assumption is rather mild in the practical applications. The requirement $d_{1}=2 d_{2}$ is not essential. It can be replaced by assuming $d_{1}>d_{2}$ and appropriately modifying the proofs in this section. We require $d_{1}>d_{2}$ to account for the fact that the transferred sources $\bar{f}_{i}$ in Algorithm 2.1 has support outside $\left(-l_{1}, l_{1}\right)$ in the $x_{1}$ direction and thus need additional damping in the $x_{1}$ direction than that in the $x_{2}$ direction.

In this section we will denote $C=C\left(\sigma_{0}, \gamma_{0}^{-1}\right)$ the generic constants which are independent of $k, d_{1}, d_{2}$ but may depend on $\sigma_{0}$ and $\gamma_{0}^{-1}$ with at most polynomial growth as $\sigma_{0}, \gamma_{0}^{-1}$ increase. As we are interested in the case of large wave number, without loss of generality, we assume $k \geq 1$ in the following.

3.1. Convergence of the PML method. The well-posedness of the truncated PML problem (3.3) is studied in [3], [12], [18] under different conditions on the medium property. We will show by adapting the argument in [3] that under the condition on medium property in (2.2), the sesquilinear form associated with (3.3) satisfies the inf-sup condition:

$$
\sup _{\psi \in H_{0}^{1}\left(B_{L}\right)} \frac{\left|(A \nabla \phi, \nabla \psi)-k^{2}(J \phi, \psi)\right|}{\|\psi\|_{H^{1}\left(B_{L}\right)}} \geq \mu_{L}\|\phi\|_{H^{1}\left(B_{L}\right)}, \quad \forall \phi \in H_{0}^{1}\left(B_{L}\right),
$$

where $\mu_{L}>0$ is a constant. The purpose of the proof is to provide a growth estimate of $\mu_{L}^{-1} \leq C k^{3 / 2}$ for some constant $C=C\left(\sigma_{0}, \gamma_{0}^{-1}\right)$ that has at most polynomial growth in $\sigma_{0}$ and $\gamma_{0}^{-1}$.

We start with the following elementary lemma.

Lemma 3.1. Let $\beta>1$ be a fixed number. If $|x-y| \geq 2 \sqrt{2} \beta M$, where $M=$ $\max \left(\bar{l}_{1}, \bar{l}_{2}\right)$, we have $\operatorname{Im} \rho(\tilde{x}, \tilde{y}) \geq \frac{1}{2} \frac{\beta-1}{\beta} \gamma_{0}|x-y|$. 
Proof. Let $j$ be the index such that $\left|x_{j}-y_{j}\right|=\max \left(\left|x_{1}-y_{1}\right|,\left|x_{2}-y_{2}\right|\right)$. Then $\left|x_{j}-y_{j}\right|^{2} \geq|x-y|^{2} / 2$. Moreover, it follows from the assumption $|x-y| \geq 2 \sqrt{2} \beta M$ that $\left|x_{j}-y_{j}\right| \geq 2 \beta \bar{l}_{j}$. Thus, since $\sigma(t)=\gamma_{0}$ for $|t| \geq \bar{l}_{j}$,

$$
\frac{1}{\left|x_{j}-y_{j}\right|}\left|\int_{y_{j}}^{x_{j}} \sigma(t) d t\right| \geq \frac{\left(\left|x_{j}-y_{j}\right|-2 \bar{l}_{j}\right) \gamma_{0}}{\left|x_{j}-y_{j}\right|} \geq \frac{\beta-1}{\beta} \gamma_{0} .
$$

This implies by (2.5) that

$$
\operatorname{Im} \rho(\tilde{x}, \tilde{y}) \geq \frac{\left|x_{j}-y_{j}\right|^{2}}{|x-y|} \frac{\beta-1}{\beta} \gamma_{0} \geq \frac{1}{2} \frac{\beta-1}{\beta} \gamma_{0}|x-y| .
$$

This completes the proof.

LEMma 3.2. We have

$$
\max _{x \in \mathbb{R}^{2}} \int_{\mathbb{R}^{2}}|G(\tilde{x}, \tilde{y})| d y \leq C k^{-1 / 2}, \max _{x \in \mathbb{R}^{2}} \int_{\mathbb{R}^{2}}\left|\nabla_{x} G(\tilde{x}, \tilde{y})\right| d y \leq C k^{1 / 2} .
$$

Proof. We only prove the first estimate. The second estimate can be proved similarly. By Lemma 2.5 we know that

$$
\begin{aligned}
\int_{\mathbb{R}^{2}}\left|H_{0}^{(1)}(k \rho(\tilde{x}, \tilde{y}))\right| d y & \leq C k^{-1 / 2}\left(\int_{|x-y|<\beta_{1}} \frac{1}{|x-y|^{1 / 2}} d y+\int_{|x-y|>\beta_{1}} \frac{e^{-\frac{1}{2} k \zeta|x-y|}}{|x-y|^{1 / 2}} d y\right) \\
& \leq C k^{-1 / 2},
\end{aligned}
$$

where $\beta_{1}=2 \sqrt{2} \beta M$ and $\zeta=\frac{1}{2} \frac{\beta-1}{\beta} \gamma_{0}$. This completes the proof.

The first step to prove the inf-sup condition for the truncated PML problem is the stability of the PML problem in $\mathbb{R}^{2}$.

Lemma 3.3. For any $F \in H^{1}\left(\mathbb{R}^{2}\right)^{\prime}$, there exists a unique solution $w \in H^{1}\left(\mathbb{R}^{2}\right)$ of the problem

$$
(A \nabla w, \nabla \psi)-k^{2}(J w, \psi)=\langle F, \psi\rangle, \quad \forall \psi \in H^{1}\left(\mathbb{R}^{2}\right) .
$$

Moreover, we have $\|w\|_{H^{1}\left(\mathbb{R}^{2}\right)} \leq C k^{3 / 2}\|F\|_{H^{1}\left(\mathbb{R}^{2}\right)^{\prime}}$.

Proof. It is known [18] that (3.5) has a unique solution under the condition of medium property imposed in (2.2). We recall briefly the argument in [3] to track the dependence of the stability constant on $k, \sigma_{0}, \gamma_{0}^{-1}$. Since $\operatorname{Re} \frac{\alpha_{1}\left(x_{1}\right)}{\alpha_{2}\left(x_{2}\right)} \geq \frac{1}{1+\sigma_{0}^{2}}$ and $\operatorname{Re} \frac{\alpha_{2}\left(x_{2}\right)}{\alpha_{1}\left(x_{1}\right)} \geq \frac{1}{1+\sigma_{0}^{2}}$, for any $F \in H^{1}\left(\mathbb{R}^{2}\right)^{\prime}$, by Lax-Milgram lemma we know that there exists a unique solution $v \in H^{1}\left(\mathbb{R}^{2}\right)$ of the problem

$$
(A \nabla v, \nabla \psi)+k^{2}(v, \psi)=\langle F, \psi\rangle, \quad \forall \psi \in H^{1}\left(\mathbb{R}^{2}\right) .
$$

Moreover, $\|v\|_{H^{1}\left(\mathbb{R}^{2}\right)} \leq C\|F\|_{H^{1}\left(\mathbb{R}^{2}\right)^{\prime}}$. For $F_{1}=k^{2} J^{-1} v+k^{2} v \in L^{2}\left(\mathbb{R}^{2}\right)$, we introduce the Newton potential

$$
\Phi(x)=\int_{\mathbb{R}^{2}} J(y) G(\tilde{x}, \tilde{y}) F_{1}(y) d y, \quad \forall x \in \mathbb{R}^{2},
$$

which, since $\tilde{G}(x, y)=J(y) G(\tilde{x}, \tilde{y})$ is the fundamental solution of the PML equation, satisfies

$$
(A \nabla \Phi, \nabla \psi)-k^{2}(J \Phi, \psi)=\left(J F_{1}, \psi\right), \quad \forall \psi \in H^{1}\left(\mathbb{R}^{2}\right)
$$


It is then clear that $w=v+\Phi$ satisfies (3.5). It remains to show

$$
\|\Phi\|_{H^{1}\left(\mathbb{R}^{2}\right)} \leq C k^{1 / 2}\left\|F_{1}\right\|_{L^{2}\left(\mathbb{R}^{2}\right)}
$$

to complete the proof since $\left\|F_{1}\right\|_{L^{2}\left(\mathbb{R}^{2}\right)} \leq C k^{2}\|v\|_{L^{2}\left(\mathbb{R}^{2}\right)} \leq C k\|F\|_{H^{1}\left(\mathbb{R}^{2}\right)^{\prime}}$. To show (3.9), we notice that for any $g \in L^{2}\left(\mathbb{R}^{2}\right)$, by Cauchy-Schwarz inequality,

$$
\begin{aligned}
|(\Phi, g)| & =\left|\int_{\mathbb{R}^{2}} \int_{\mathbb{R}^{2}} J(y) G(\tilde{x}, \tilde{y}) F_{1}(y) g(x) d x d y\right| \\
& \leq C\left(\int_{\mathbb{R}^{2}} \int_{\mathbb{R}^{2}}\left|G(\tilde{x}, \tilde{y}) \| F_{1}(y)\right|^{2} d x d y\right)^{1 / 2}\left(\int_{\mathbb{R}^{2}} \int_{\mathbb{R}^{2}}|G(\tilde{x}, \tilde{y}) \| g(x)|^{2} d x d y\right)^{1 / 2} \\
& \leq C \max _{x \in \mathbb{R}^{2}}\left(\int_{\mathbb{R}^{2}}|G(\tilde{x}, \tilde{y})| d y\right)\left\|F_{1}\right\|_{L^{2}\left(\mathbb{R}^{2}\right)}\|g\|_{L^{2}\left(\mathbb{R}^{2}\right)} .
\end{aligned}
$$

This implies $\|\Phi\|_{L^{2}\left(\mathbb{R}^{2}\right)} \leq C k^{-1 / 2}\left\|F_{1}\right\|_{L^{2}\left(\mathbb{R}^{2}\right)}$ by Lemma 3.2. Similarly, one can show $\|\nabla \Phi\|_{L^{2}\left(\mathbb{R}^{2}\right)} \leq C k^{1 / 2}\left\|F_{1}\right\|_{L^{2}\left(\mathbb{R}^{2}\right)}$. This concludes the proof.

The above lemma indicates the following inf-sup condition for the sesquiliner form associated with the PML problem in $\mathbb{R}^{2}$ :

$$
\sup _{\psi \in H^{1}\left(\mathbb{R}^{2}\right)} \frac{\left|(A \nabla \phi, \nabla \psi)-k^{2}(J \phi, \psi)\right|}{\|\psi\|_{H^{1}\left(\mathbb{R}^{2}\right)}} \geq \mu_{0}\|\phi\|_{H^{1}\left(\mathbb{R}^{2}\right)}, \quad \forall \phi \in H^{1}\left(\mathbb{R}^{2}\right) .
$$

The inf-sup constant $\mu_{0}$ satisfies $\mu_{0}^{-1} \leq C k^{3 / 2}$.

LEMma 3.4. For sufficiently large $\sigma_{0} d_{2} \geq 1$, the sesquilinear form associated with (3.3) satisfies the inf-sup condition (3.4) with the constant $\mu_{L}^{-1} \leq C k^{3 / 2}$. Moreover, we have

$$
\|\tilde{u}-\hat{u}\|_{H^{1}\left(B_{L}\right)} \leq C k^{2}(1+k L)^{2} e^{-\frac{1}{2} k \gamma \bar{\sigma}}\|f\|_{H^{1}\left(B_{l}\right)^{\prime}},
$$

where $L$ is the diameter of $B_{L}$ and $\gamma=\frac{d_{2}}{\sqrt{d_{2}^{2}+\left(2 l_{2}+d_{1}+d_{2}\right)^{2}}}$. Here $\tilde{u}$ is defined in (2.9) and $\hat{u}$ is the solution of the problem (3.3).

Proof. By the reflection argument in [3, Theorem 5.5], one can show (3.4) in the bounded domain $B_{L}$ for sufficiently large $\sigma_{0} d_{2}$ with the inf-sup constant $\mu_{L}=$ $\mu_{0} / 8$. This implies $\mu_{L}^{-1} \leq C k^{3 / 2}$. Here we briefly recall the argument to track the dependence of the constant $\mu_{L}$ on $\mu_{0}$. For any $\phi \in H_{0}^{1}\left(B_{L}\right)$, define $F \in H^{-1}\left(B_{L}\right)$ by

$$
\langle F, \psi\rangle=(A \nabla \phi, \nabla \psi)-k^{2}(J \phi, \psi), \quad \forall \psi \in H_{0}^{1}\left(B_{L}\right) .
$$

Then the inf-sup condition (3.4) is equivalent to show $\|\phi\|_{H^{1}\left(B_{L}\right)} \leq \mu_{L}^{-1}\|F\|_{H^{-1}\left(B_{L}\right)}$. Denote by $L_{j}=l_{j}+d_{j}$ and $B_{L}^{R}=\left(-2 L_{1}+\bar{l}_{1}, 2 L_{1}-\bar{l}_{1}\right) \times\left(-2 L_{2}+\bar{l}_{2}, 2 L_{2}-\bar{l}_{2}\right)$. We define $\tilde{\phi} \in H^{1}\left(B_{L}^{R}\right)$ as follows. First let

$$
\phi_{1}(x)= \begin{cases}-\phi\left(2 L_{1}-x_{1}, x_{2}\right) & \text { if } x \in\left(L_{1}, 2 L_{1}-\bar{l}_{1}\right) \times\left(-L_{2}, L_{2}\right) ; \\ \phi\left(x_{1}, x_{2}\right) & \text { if } x \in\left(-L_{1}, L_{1}\right) \times\left(-L_{2}, L_{2}\right) \\ -\phi\left(-2 L_{1}-x_{1}, x_{2}\right) & \text { if } x \in\left(-2 L_{1}+\bar{l}_{1},-L_{1}\right) \times\left(-L_{2}, L_{2}\right) .\end{cases}
$$

$\phi_{1}$ is the extension of $\phi$ to $\left(-2 L_{1}+\bar{l}_{1}, 2 L_{1}-\bar{l}_{1}\right) \times\left(-L_{2}, L_{2}\right)$ by odd reflection with respect to $x_{1}= \pm L_{1}$. Then $\tilde{\phi}$ is defined as the extension of $\phi_{1}$ to $B_{L}^{R}$ by odd reflection with respect to $x_{1}= \pm L_{2}$

$$
\tilde{\phi}(x)= \begin{cases}-\phi_{1}\left(x_{1}, 2 L_{2}-x_{2}\right) & \text { if } x \in\left(-2 L_{1}+\bar{l}_{1}, 2 L_{1}-\bar{l}_{1}\right) \times\left(L_{2}, 2 L_{2}-\bar{l}_{2}\right) ; \\ \phi_{1}\left(x_{1}, x_{2}\right) & \text { if } x \in\left(-2 L_{1}+\bar{l}_{1}, 2 L_{1}-\bar{l}_{1}\right) \times\left(-L_{2}, L_{2}\right) ; \\ -\phi_{1}\left(x_{1},-2 L_{2}-x_{2}\right) & \text { if } x \in\left(-2 L_{1}+\bar{l}_{1}, 2 L_{1}-\bar{l}_{1}\right) \times\left(-2 L_{2}+\bar{l}_{2},-L_{2}\right) .\end{cases}
$$


Now we define the functional $\tilde{F} \in H^{-1}\left(B_{L}^{R}\right)$ by

$$
\langle\tilde{F}, \psi\rangle=(A \nabla \tilde{\phi}, \nabla \psi)-k^{2}(J \tilde{\phi}, \psi), \quad \forall \psi \in H_{0}^{1}\left(B_{L}^{R}\right) .
$$

It is clear that $\tilde{F}$ is an extension of $F \in H^{-1}\left(B_{L}\right)$ and since $\sigma_{j}=\gamma_{0}$ for $|t| \geq$ $\bar{l}_{j}$, we know that $\|\tilde{F}\|_{H^{-1}\left(B_{L}^{R}\right)} \leq 4\|F\|_{H^{-1}\left(B_{L}\right)}$. Now we extend $\tilde{F}$ to a functional $F_{1} \in H^{1}\left(\mathbb{R}^{2}\right)^{\prime}$ by Hahn-Banach theorem such that $\left\|F_{1}\right\|_{H^{1}\left(\mathbb{R}^{2}\right)^{\prime}}=\|\tilde{F}\|_{H^{-1}\left(B_{L}^{R}\right)} \leq$ $4\|F\|_{H^{-1}\left(B_{L}\right)}$. For $F_{1} \in H^{1}\left(\mathbb{R}^{2}\right)^{\prime}$ we use Lemma 3.3 and the inf-sup condition (3.10) to conclude that there exists a $v \in H^{1}\left(\mathbb{R}^{2}\right)$ such that

$$
(A \nabla v, \nabla \psi)-k^{2}(J v, \psi)=\left\langle F_{1}, \psi\right\rangle, \quad \forall \psi \in H^{1}\left(\mathbb{R}^{2}\right),
$$

and satisfies $\|v\|_{H^{1}\left(\mathbb{R}^{2}\right)} \leq \mu_{0}^{-1}\left\|F_{1}\right\|_{H^{1}\left(\mathbb{R}^{2}\right)^{\prime}} \leq 4 \mu_{0}^{-1}\|F\|_{H^{-1}\left(B_{L}\right)}$. Thus

$$
\|\phi\|_{H^{1}\left(B_{L}\right)} \leq\|v\|_{H^{1}\left(B_{L}\right)}+\|v-\tilde{\phi}\|_{H^{1}\left(B_{L}\right)} \leq 4 \mu_{0}^{-1}\|F\|_{H^{-1}\left(B_{L}\right)}+\|v-\tilde{\phi}\|_{H^{1}\left(B_{L}\right)} .
$$

It is easy to see that $v-\tilde{\phi} \in H^{1}\left(B_{L}^{R}\right)$ satisfies the homogeneous PML equation

$$
(A \nabla(v-\tilde{\phi}), \nabla \psi)-k^{2}(J(v-\tilde{\phi}), \psi)=0, \quad \forall \psi \in H_{0}^{1}\left(B_{L}^{R}\right) .
$$

From the proof in $\left[3\right.$, Theorem 5.5] one can show that $\|v-\tilde{\phi}\|_{H^{1}\left(B_{L}\right)}$ decays exponentially and can be bounded by $\frac{1}{2}\|\phi\|_{H^{1}\left(B_{L}\right)}$ for sufficiently large $\sigma_{0} d_{2}$. This shows the inf-sup condition (3.4) with $\mu_{L}=\mu_{0} / 8$.

Now we prove the error estimate. Let $\tilde{u} \in H^{1}\left(\mathbb{R}^{2}\right)$ be defined in (2.9) which satisfies the PML equation (2.10). It is clear that its trace $\tilde{u} \in H^{1 / 2}\left(\partial B_{L}\right)$. By (3.2), there exists a lifting function $\phi \in H^{1}\left(B_{L}\right)$ such that $\tilde{u}=\phi$ on $\partial B_{L}$ and $\|\phi\|_{H^{1}\left(B_{L}\right)} \leq$ $C\|\tilde{u}\|_{H^{1 / 2}\left(\partial B_{L}\right)}$. Since $\tilde{u}-\hat{u}-\phi \in H_{0}^{1}\left(B_{L}\right)$, by (3.4) and the equations (2.10) and (3.3) satisfied by $\tilde{u}$ and $\hat{u}$, we obtain

$$
\|\tilde{u}-\hat{u}-\phi\|_{H^{1}\left(B_{L}\right)} \leq \mu_{L}^{-1} \sup _{\psi \in H_{0}^{1}\left(B_{L}\right)} \frac{\left|(A \nabla \phi, \nabla \psi)-k^{2}(J \phi, \psi)\right|}{\|\psi\|_{H^{1}\left(B_{L}\right)}} \leq C \mu_{L}^{-1}\|\phi\|_{H^{1}\left(B_{L}\right)} .
$$

This implies by (3.1) that

$$
\begin{aligned}
\|\tilde{u}-\hat{u}\|_{H^{1}\left(B_{L}\right)} \leq C\left(1+\mu_{L}^{-1}\right)\|\phi\|_{H^{1}\left(B_{L}\right)} & \leq C k^{3 / 2}(1+k L)\|\phi\|_{H^{1}\left(B_{L}\right)} \\
& \leq C k^{3 / 2}(1+k L)\|\tilde{u}\|_{H^{1 / 2}\left(\partial B_{L}\right)} \\
& \leq C k^{3 / 2}(1+k L) \max _{x \in \partial B_{L}}(|\tilde{u}(x)|+L|\nabla \tilde{u}(x)|) .
\end{aligned}
$$

By (2.9) and Lemma 2.5, since $|x-y| \geq d_{2}$ for $x \in \partial B_{L}, y \in B_{l}$, we have

$$
\begin{aligned}
|\tilde{u}(x)| & \leq C\|f\|_{H^{1}\left(B_{l}\right)^{\prime}} \max _{y \in B_{l}}\left(k|G(\tilde{x}, \tilde{y})|+\left|\nabla_{y} G(\tilde{x}, \tilde{y})\right|\right) \\
& \leq C e^{-\frac{1}{2} k \operatorname{Im} \rho(\tilde{x}, \tilde{y})}\left[k\left(k d_{2}\right)^{-1 / 2}+k\left(\left(k d_{2}\right)^{-1 / 2}+\left(k d_{2}\right)^{-1}\right)\right]\|f\|_{H^{1}\left(B_{l}\right)^{\prime}} \\
& \leq C k^{1 / 2} e^{-\frac{1}{2} k \operatorname{Im} \rho(\tilde{x}, \tilde{y})}\|f\|_{H^{1}\left(B_{l}\right)^{\prime}}, \quad \forall x \in \partial B_{L},
\end{aligned}
$$

where the constant $C$ is independent of $d_{2}$ since $d_{2}>\bar{l}_{2}-l_{2}$ by the construction of PML layer and $\bar{l}_{2}$ is the fixed constant introduced in (2.2). Similarly, one can show that for any $x \in \partial B_{L},|\nabla \tilde{u}(x)| \leq C k^{3 / 2} e^{-\frac{1}{2} k \operatorname{Im} \rho(\tilde{x}, \tilde{y})}\|f\|_{H^{1}\left(B_{l}\right)^{\prime}}$. Therefore,

$$
\|\tilde{u}-\hat{u}\|_{H^{1}\left(B_{L}\right)} \leq C k^{2}(1+k L)^{2} e^{-\frac{1}{2} k \operatorname{Im} \rho(\tilde{x}, \tilde{y})}\|f\|_{H^{1}\left(B_{l}\right)^{\prime}} .
$$


On the other hand, for $x \in \partial B_{L}, y \in B_{l}$, we have

$$
\frac{\left|x_{i}-y_{i}\right|}{|x-y|} \geq \min \left(\frac{d_{1}}{\sqrt{d_{1}^{2}+\left(2 l_{2}+d_{2}\right)^{2}}}, \frac{d_{2}}{\sqrt{d_{2}^{2}+\left(2 l_{1}+d_{1}\right)^{2}}}\right) \geq \gamma, \quad i=1,2,
$$

where we have used the assumption $l_{1} \leq l_{2}$ of (H1) in the last inequality. Now we deduce from (2.5) and (H1) that

$$
\operatorname{Im} \rho(\tilde{x}, \tilde{y}) \geq \min _{j=1,2} \frac{\left|x_{j}-y_{j}\right|}{|x-y|}\left|\int_{y_{j}}^{x_{j}} \sigma_{j}(t) d t\right| \geq \gamma \bar{\sigma}, \quad \forall x \in \partial B_{L}, y \in B_{l} .
$$

This completes the proof. $\square$

3.2. Truncation of the source transfer PML problems. The key step in the source transfer algorithm in $\mathbb{R}^{2}$, Algorithm 2.1, is solve (2.15) whose source is supported in $\Omega_{i}$ and we need the solution $u_{i}$ in $\Omega_{i+1}$. Therefore, we will solve (2.15) by putting PML outside the domain $\left(-l_{1}, l_{1}\right) \times\left(\zeta_{i}, \zeta_{i+2}\right)$. The PML complex coordinate stretching outside the domain $\left(-l_{1}, l_{1}\right) \times\left(\zeta_{i}, \zeta_{i+2}\right)$ is $\tilde{x}_{i}(x)=\left(\tilde{x}_{i, 1}\left(x_{1}\right), \tilde{x}_{i, 2}\left(x_{2}\right)\right)^{T}$, where $\tilde{x}_{i, 1}\left(x_{1}\right)=\tilde{x}_{1}\left(x_{1}\right)$ and

$$
\tilde{x}_{i, 2}\left(x_{2}\right)= \begin{cases}x_{2}+\mathbf{i} \int_{\zeta_{i+2}}^{x_{2}} \sigma_{2}\left(t+\zeta_{N+1}-\zeta_{i+2}\right) d t & \text { if } x_{2}>\zeta_{i+2}, \\ x_{2} & \text { if } \zeta_{i} \leq x_{2} \leq \zeta_{i+2}, \\ x_{2}+\mathbf{i} \int_{\zeta_{i}}^{x_{2}} \sigma_{2}\left(t-\zeta_{i}+\zeta_{1}\right) d t & \text { if } x_{2}<\zeta_{i} .\end{cases}
$$

We denote $A_{i}(x)=\operatorname{diag}\left(\frac{\tilde{x}_{i, 2}\left(x_{2}\right)^{\prime}}{\tilde{x}_{i, 1}\left(x_{1}\right)^{\prime}}, \frac{\tilde{x}_{i, 1}\left(x_{1}\right)^{\prime}}{\tilde{x}_{i, 2}\left(x_{2}\right)^{\prime}}\right)$ and $J_{i}(x)=\tilde{x}_{i, 1}\left(x_{1}\right)^{\prime} \tilde{x}_{i, 2}\left(x_{2}\right)^{\prime}$. Let

$$
\bar{u}_{i}(x)=\int_{\Omega_{i}} \bar{f}_{i}(y) J_{i}(y) G\left(\tilde{x}_{i}, \tilde{y}_{i}\right) d y, \quad i=1, \cdots, N-2 .
$$

By (2.16) and the fact that $\tilde{x}_{i}(x)=\tilde{x}(x)$ in $\Omega_{i} \cup \Omega_{i+1}$ and $\tilde{y}_{i}(y)=\tilde{y}(y)$ in $\Omega_{i}$, we have $\bar{u}_{i}=u_{i}$ in $\Omega_{i} \cup \Omega_{i+1}, i=1, \cdots, N-2$. From the definition of Algorithm 2.1, we know that, since $A_{i}=A, J_{i}=J$ in $\Omega_{i+1}$,

$$
\Psi_{i+1}\left(\bar{f}_{i}\right)(x)=J_{i}^{-1} \nabla \cdot\left(A_{i} \nabla\left(\beta_{i+1} \bar{u}_{i}\right)\right)+k^{2}\left(\beta_{i+1} \bar{u}_{i}\right) \quad \text { in } \Omega_{i+1} .
$$

This motivates the following source transfer algorithm for the truncated PML problem.

Algorithm 3.1. (Source transfer for truncated PML problem)

$1^{\circ}$ Let $\hat{f}_{1}=f_{1}$ in $\Omega_{1}$;

$2^{\circ}$ While $i=1, \cdots, N-2$, do

- Find $\hat{u}_{i} \in H_{0}^{1}\left(\Omega_{i}^{\mathrm{PML}}\right)$, where $\Omega_{i}^{\mathrm{PML}}=\left(-l_{1}-d_{1}, l_{1}+d_{1}\right) \times\left(\zeta_{i}-d_{2}, \zeta_{i+2}+d_{2}\right)$, such that

$$
\left(A_{i} \nabla \hat{u}_{i}, \nabla \psi\right)-k^{2}\left(J_{i} \hat{u}_{i}, \psi\right)=\left\langle J_{i} \hat{f}_{i}, \psi\right\rangle, \quad \forall \psi \in H_{0}^{1}\left(\Omega_{i}^{\mathrm{PML}}\right) .
$$

- Compute $\hat{\Psi}_{i+1}\left(\hat{f}_{i}\right) \in H^{-1}\left(\Omega_{i}^{\mathrm{PML}}\right)$ such that

$$
\left\langle J_{i} \hat{\Psi}_{i+1}\left(\hat{f}_{i}\right), \psi\right\rangle=-\left(A_{i} \nabla\left(\beta_{i+1} \hat{u}_{i}\right), \nabla \psi\right)+k^{2}\left(J_{i} \beta_{i+1} \hat{u}_{i}, \psi\right), \quad \forall \psi \in H_{0}^{1}\left(\Omega_{i}^{\mathrm{PML}}\right) .
$$

- Set $\hat{f}_{i+1}=f_{i+1}+\hat{\Psi}_{i+1}\left(\hat{f}_{i}\right)$ in $\Omega_{i+1} \cap B_{L}$ and $\hat{f}_{i+1}=0$ elsewhere. 


\section{End while}

By the same argument in Section 3.1 for the PML problem (3.3), we can show that for sufficiently large $\sigma_{0} d_{2}$, the sesquilinear form associated with (3.15) satisfies the following inf-sup condition:

$$
\sup _{\psi \in H_{0}^{1}\left(\Omega_{i}^{\mathrm{PML}}\right)} \frac{\left|\left(A_{i} \nabla \phi, \nabla \psi\right)-k^{2}\left(J_{i} \phi, \psi\right)\right|}{\|\psi\|_{H^{1}\left(\Omega_{i}^{\mathrm{PML}}\right)}} \geq \mu_{i}\|\phi\|_{H^{1}\left(\Omega_{i}^{\mathrm{PML}}\right)}, \quad \forall \phi \in H_{0}^{1}\left(\Omega_{i}^{\mathrm{PML}}\right) .
$$

The inf-sup constant $\mu_{i}$ satisfies $\mu_{i}^{-1} \leq C k^{3 / 2}$. Here we omit the details.

Lemma 3.5. Let $\sigma_{0} d_{2} \geq 1$ be sufficiently large. For $i=1, \cdots, N-2$, we have for any $x \in \Omega_{i+1}^{+}=\left\{x=\left(x_{1}, x_{2}\right)^{T} \in \Omega_{i+1}:\left|x_{1}\right|>l_{1}+d_{2}\right\}$,

$$
\left|\bar{u}_{i}(x)\right| \leq C k^{1 / 2} e^{-\frac{1}{2} k \gamma \bar{\sigma}}\|f\|_{H^{1}\left(B_{l}\right)^{\prime}}, \quad\left|\nabla \bar{u}_{i}(x)\right| \leq C k^{3 / 2} e^{-\frac{1}{2} k \gamma \bar{\sigma}}\|f\|_{H^{1}\left(B_{l}\right)^{\prime}} .
$$

Proof. By the argument in the proof of the first part of Theorem 2.7 we know that for $x \in \Omega_{i+1}$,

$$
\int_{\Omega_{i}} \Psi_{i}\left(\bar{f}_{i-1}\right)(y) J_{i}(y) G\left(\tilde{x}_{i}, \tilde{y}_{i}\right) d y=\int_{\Omega_{i-1}} \bar{f}_{i-1}(y) J_{i-1}(y) G\left(\tilde{x}_{i}, \tilde{y}_{i}\right) d y,
$$

where $\tilde{y}_{i}=\tilde{y}_{i}(y)$ is defined as that for $\tilde{x}_{i}=\tilde{x}_{i}(x)$ at the beginning of this subsection. This implies that for $x \in \Omega_{i+1}$,

$$
\bar{u}_{i}(x)=\int_{\Omega\left(\zeta_{1}, \zeta_{i+1}\right)} f(y) J(y) G(\tilde{x}, \tilde{y}) d y,
$$

where we have used that $J_{j}=1, \tilde{y}_{j}(y)=y$ in $\Omega_{j} \cap B_{l}$ for $j=1, \cdots, i$. Now for $x \in \Omega_{i+1}^{+}, y \in B_{l}$, we have $\left|x_{1}-y_{1}\right| \geq d_{2}$ and

$$
\frac{\left|x_{1}-y_{1}\right|}{|x-y|} \geq \frac{d_{2}}{\sqrt{d_{2}^{2}+\left(2 \Delta \zeta+d_{2}\right)^{2}}} \geq \frac{d_{2}}{\sqrt{d_{2}^{2}+\left(2 l_{2}+d_{2}\right)^{2}}} \geq \gamma
$$

Thus a similar argument as in (3.11) and using (H1) indicates that for $x \in \Omega_{i+1}^{+}$, $y \in B_{l},\left|\bar{u}_{i}(x)\right| \leq C k^{1 / 2} e^{-\frac{1}{2} k \gamma \bar{\sigma}}\|f\|_{H^{1}\left(B_{l}\right)^{\prime}}$. The proof of the estimate for $\left|\nabla \bar{u}_{i}(x)\right|$ is similar. This completes the proof.

Lemma 3.6. Let $\sigma_{0} d_{2} \geq 1$ be sufficiently large. For $i=1, \cdots, N-2$, we have

$$
\left\|\bar{u}_{i}\right\|_{H^{1 / 2}\left(\partial \Omega_{i}^{\mathrm{PML}}\right)} \leq C k(1+k L) e^{-\frac{1}{2} k \gamma \bar{\sigma}}\|f\|_{H^{1}\left(B_{l}\right)^{\prime}} .
$$

Proof. By (3.1) we have

$$
\left\|\bar{u}_{i}\right\|_{H^{1 / 2}\left(\partial \Omega_{i}^{\mathrm{PML}}\right)} \leq C \max _{x \in \partial \Omega_{i}^{\mathrm{PML}}}\left(\left|\bar{u}_{i}(x)\right|+L\left|\nabla \bar{u}_{i}(x)\right|\right) .
$$

Since $\bar{u}_{i-1}=u_{i-1}$ in $\Omega_{i}$, by Lemma 2.6, $\left|\bar{u}_{i-1}(y)\right|$ and $\left|\frac{\partial \bar{u}_{i-1}(y)}{\partial y_{2}}\right|$ decay exponentially in $\Omega_{i}$ as $|y| \rightarrow \infty$. Thus by using (2.17) and integration by parts, we know that

$$
\begin{aligned}
\bar{u}_{i}(x)= & \int_{\Omega_{i}} f_{i}(y) J_{i}(y) G\left(\tilde{x}_{i}, \tilde{y}_{i}\right) d y-\int_{\Omega_{i}} \frac{\alpha_{1}\left(y_{1}\right)}{\alpha_{2}\left(y_{2}\right)} \beta_{i}^{\prime}\left(y_{2}\right) \bar{u}_{i-1}(y) \frac{\partial G\left(\tilde{x}_{i}, \tilde{y}_{i}\right)}{\partial y_{2}} d y \\
& +\int_{\Omega_{i}} \beta_{i}^{\prime}\left(y_{2}\right) \frac{\alpha_{1}\left(y_{1}\right)}{\alpha_{2}\left(y_{2}\right)} \frac{\partial \bar{u}_{i-1}(y)}{\partial y_{2}} G\left(\tilde{x}_{i}, \tilde{y}_{i}\right) d y=: \bar{u}_{i}^{\mathrm{I}}+\bar{u}_{i}^{\mathrm{II}}+\bar{u}_{i}^{\mathrm{III}} .
\end{aligned}
$$


It is clear that

$$
\left|\bar{u}_{i}^{\mathrm{II}}(x)\right| \leq C \int_{\Omega_{i}^{-}}\left|\bar{u}_{i-1}(y)\right|\left|\nabla_{y} G\left(\tilde{x}_{i}, \tilde{y}_{i}\right)\right| d y+C \int_{\Omega_{i}^{+}}\left|\bar{u}_{i-1}(y)\right|\left|\nabla_{y} G\left(\tilde{x}_{i}, \tilde{y}_{i}\right)\right| d y,
$$

where $\Omega_{i}^{-}=\left\{y=\left(y_{1}, y_{2}\right)^{T} \in \Omega_{i}:\left|y_{1}\right|<l_{1}+d_{2}\right\}$ and $\Omega_{i}^{+}=\left\{y=\left(y_{1}, y_{2}\right)^{T} \in \Omega_{i}\right.$ : $\left.\left|y_{1}\right|>l_{1}+d_{2}\right\}$. Here we have used the assumption $\left|\beta_{i}^{\prime}\right| \leq C(\Delta \zeta)^{-1}$ in (2.14) in above inequality. Since by (3.17), $\bar{u}_{i-1}$ satisfies $(3.5)$ with $F(y)=f(y) \chi_{\Omega\left(\zeta_{1}, \zeta_{i}\right)}(y)$, where $\chi_{\Omega\left(\zeta_{1}, \zeta_{i}\right)}$ is the characteristic function of $\Omega\left(\zeta_{1}, \zeta_{i}\right)$, we know from Lemma 3.3 that $\left\|\bar{u}_{i-1}\right\|_{H^{1}\left(\mathbb{R}^{2}\right)} \leq C k^{3 / 2}\|f\|_{H^{1}\left(B_{l}\right)^{\prime}}$. Thus

$$
\int_{\Omega_{i}^{-}}\left|\bar{u}_{i-1}(y)\right|\left|\nabla_{y} G\left(\tilde{x}_{i}, \tilde{y}_{i}\right)\right| d y \leq C k^{1 / 2}\|f\|_{H^{1}\left(B_{l}\right)^{\prime}}\left(\int_{\Omega_{i}^{-}}\left|\nabla_{y} G\left(\tilde{x}_{i}, \tilde{y}_{i}\right)\right|^{2} d y\right)^{1 / 2} .
$$

For $x \in \partial \Omega_{i}^{\mathrm{PML}}$ and $y \in \Omega_{i}^{-}$we have either $\left|x_{1}-y_{1}\right| \geq d_{2}$ or $\left|x_{2}-y_{2}\right| \geq d_{2}$, by (2.5) and (H1)

$$
\operatorname{Im} \rho\left(\tilde{x}_{i}, \tilde{y}_{i}\right) \geq \min _{j=1,2} \frac{\left|x_{j}-y_{j}\right|}{|x-y|}\left|\int_{y_{j}}^{x_{j}} \sigma_{j}(t) d t\right| \geq \gamma \bar{\sigma} .
$$

Thus by Lemma 2.5

$$
\int_{\Omega_{i}^{-}}\left|\bar{u}_{i-1}(y)\right|\left|\nabla_{y} G\left(\tilde{x}_{i}, \tilde{y}_{i}\right)\right| d y \leq C k e^{-\frac{1}{2} k \gamma \bar{\sigma}}\|f\|_{H^{1}\left(B_{l}\right)^{\prime}}, \quad \forall x \in \partial \Omega_{i}^{\mathrm{PML}} .
$$

Next by Lemma 3.5 and Lemma 3.2, for $x \in \partial \Omega_{i}^{\mathrm{PML}}$, we have

$$
\begin{aligned}
\int_{\Omega_{i}^{+}}\left|\bar{u}_{i-1}(y) \| \nabla_{y} G\left(\tilde{x}_{i}, \tilde{y}_{i}\right)\right| d y & \leq C k^{1 / 2} e^{-\frac{1}{2} k \gamma \bar{\sigma}}\|f\|_{H^{1}\left(B_{l}\right)^{\prime}} \int_{\Omega_{i}^{+}}\left|\nabla_{y} G\left(\tilde{x}_{i}, \tilde{y}_{i}\right)\right| d y \\
& \leq C k e^{-\frac{1}{2} k \gamma \bar{\sigma}}\|f\|_{H^{1}\left(B_{l}\right)^{\prime}} .
\end{aligned}
$$

Therefore, we have $\left|\bar{u}_{i}^{\mathrm{II}}(x)\right| \leq C k e^{-\frac{1}{2} k \gamma \bar{\sigma}}\|f\|_{H^{1}\left(B_{l}\right)^{\prime}}$ for $x \in \partial \Omega_{i}^{\mathrm{PML}}$. A similar argument implies $\left|\bar{u}_{i}^{\mathrm{I}}(x)\right|+\left|\bar{u}_{i}^{\mathrm{III}}(x)\right| \leq C k e^{-\frac{1}{2} k \gamma \bar{\sigma}}\|f\|_{H^{1}\left(B_{l}\right)^{\prime}}$ for $x \in \partial \Omega_{i}^{\mathrm{PML}}$. This shows $\left|\bar{u}_{i}(x)\right| \leq C k e^{-\frac{1}{2} k \gamma \bar{\sigma}}\|f\|_{H^{1}\left(B_{l}\right)^{\prime}}$ for $x \in \partial \Omega_{i}^{\mathrm{PML}}$. Similarly, we have $\left|\nabla \bar{u}_{i}(x)\right| \leq$ $C k^{2} e^{-\frac{1}{2} k \gamma \bar{\sigma}}\|f\|_{H^{1}\left(B_{l}\right)^{\prime}}$ for $x \in \partial \Omega_{i}^{\mathrm{PML}}$. This completes the proof by using (3.18).

The following theorem shows that $\hat{f}_{i}$ is a good approximation of $\bar{f}_{i}$ in Algorithm 2.1 .

TheOrem 3.7. Let $\sigma_{0} d_{2} \geq 1$ be sufficiently large. For $i=2, \cdots, N-1$, we have

$$
\left\|\bar{f}_{i}-\hat{f}_{i}\right\|_{H^{-1}\left(\Omega_{i}^{\mathrm{PML}}\right)} \leq C k^{\frac{3}{2} i-\frac{1}{2}}(1+k L)^{2} e^{-\frac{1}{2} k \gamma \bar{\sigma}}\|f\|_{H^{1}\left(B_{l}\right)^{\prime}} .
$$

Proof. By definition and the calculation in (2.17) we have

$$
\begin{aligned}
\left\|\bar{f}_{i}-\hat{f}_{i}\right\|_{H^{-1}\left(\Omega_{i}^{\mathrm{PML}}\right)} & =\left\|\Psi_{i}\left(\bar{f}_{i-1}\right)-\hat{\Psi}_{i}\left(\hat{f}_{i-1}\right)\right\|_{H^{-1}\left(\Omega_{i}^{\mathrm{PML}}\right)} \\
& \leq C\left\|\bar{u}_{i-1}-\hat{u}_{i-1}\right\|_{H^{1}\left(\Omega_{i-1}^{\mathrm{PML}}\right)} .
\end{aligned}
$$

It is clear that $\bar{u}_{i-1}-\hat{u}_{i-1}$ satisfies $\bar{u}_{i-1}-\hat{u}_{i-1}=\bar{u}_{i-1}$ on $\partial \Omega_{i}^{\mathrm{PML}}$, and

$$
\left(A_{i-1} \nabla\left(\bar{u}_{i-1}-\hat{u}_{i-1}\right), \nabla \psi\right)-k^{2}\left(J_{i-1}\left(\bar{u}_{i-1}-\hat{u}_{i-1}\right), \psi\right)=\left\langle J_{i-1}\left(\bar{f}_{i-1}-\hat{f}_{i-1}\right), \psi\right\rangle,
$$


for any $\psi \in H_{0}^{1}\left(\Omega_{i-1}^{\mathrm{PML}}\right)$. This implies by (3.16) and the standard argument that

$$
\begin{aligned}
\left\|\bar{u}_{i-1}-\hat{u}_{i-1}\right\|_{H^{1}\left(\Omega_{i-1}^{\mathrm{PML}}\right)} \leq C k^{3 / 2}\left\|\bar{f}_{i-1}-\hat{f}_{i-1}\right\|_{H^{-1}\left(\Omega_{i-1}^{\mathrm{PML}}\right)} \\
+C k^{3 / 2}(1+k L)\left\|\bar{u}_{i-1}\right\|_{H^{1 / 2}\left(\partial \Omega_{i-1}^{\mathrm{PML}}\right)} .
\end{aligned}
$$

Thus by Lemma 3.6 that

$$
\begin{aligned}
\left\|\bar{f}_{i}-\hat{f}_{i}\right\|_{H^{-1}\left(\Omega_{i}^{\mathrm{PML}}\right)} & \leq C k^{3 / 2}\left\|\bar{f}_{i-1}-\hat{f}_{i-1}\right\|_{H^{-1}\left(\Omega_{i-1}^{\mathrm{PML}}\right)} \\
& +C k^{5 / 2}(1+k L)^{2} e^{-\frac{1}{2} k \gamma \bar{\sigma}}\|f\|_{H^{1}\left(B_{l}\right)^{\prime}}
\end{aligned}
$$

This completes the proof by the induction argument since $\bar{f}_{1}-\hat{f}_{1}=0$.

3.3. Truncation of the wave expansion PML problems. To introduce the wave expansion algorithm for the truncated PML problem, we need to define the PML truncation of (2.19) and (2.20)-(2.21). The PML layer for (2.19) is put outside the domain $\left(-l_{1}, l_{1}\right) \times\left(\zeta_{N-1}, \zeta_{N+1}\right)$. The problem (2.20)-(2.21) is a half-space problem for which the PML layer is put outside the domain $\left(-l_{1}, l_{1}\right) \times\left(\zeta_{i-1}, \zeta_{i+1}\right)$ in the half-space $\Omega\left(-\infty, \zeta_{i+1}\right)$.

Algorithm 3.2. (WaVe EXPansion For truncated PML Problem)

$1^{\circ}$ Find $\hat{v}_{N} \in H_{0}^{1}\left(\Omega_{N-1}^{\mathrm{PML}}\right)$, where $\Omega_{N-1}^{\mathrm{PML}}=\left(-l_{1}-d_{1}, l_{1}+d_{1}\right) \times\left(\zeta_{N-1}-d_{2}, \zeta_{N+1}+d_{2}\right)$, such that

$$
\left(A_{N-1} \nabla \hat{v}_{N}, \nabla \psi\right)-k^{2}\left(J_{N-1} \hat{v}_{N}, \psi\right)=-\left\langle J_{N-1}\left(f_{N}+\hat{f}_{N-1}\right), \psi\right\rangle, \quad \forall \psi \in H_{0}^{1}\left(\Omega_{N-1}^{\mathrm{PML}}\right) .
$$

$2^{\circ}$ For $i=N-1, \cdots, 2$, find $\hat{v}_{i} \in H^{1}\left(D_{i}^{\mathrm{PML}}\right)$, where $D_{i}^{\mathrm{PML}}=\left(-l_{1}-d_{1}, l_{1}+d_{1}\right) \times$ $\left(\zeta_{i-1}-d_{2}, \zeta_{i+1}\right)$, such that $\hat{v}_{i}=\hat{v}_{i+1}$ on $\partial D_{i}^{\mathrm{PML}} \cap \Gamma_{i+1}, \hat{v}_{i}=0$ on $\partial D_{i}^{\mathrm{PML}} \backslash \Gamma_{i+1}$, and

$$
\left(A_{i-1} \nabla \hat{v}_{i}, \nabla \psi\right)-k^{2}\left(J_{i-1} \hat{v}_{i}, \psi\right)=-\left\langle J_{i-1}\left(f_{i}+\hat{f}_{i-1}\right), \psi\right\rangle, \quad \forall \psi \in H_{0}^{1}\left(D_{i}^{\mathrm{PML}}\right) .
$$

The truncated half-space PML problem in the second step of Algorithm 3.2 can be studied by the method in $[18,3]$. We remark that since the half-space Green function $\Phi_{i+1}(x, y)=\tilde{G}_{i+1}^{R}(x, y)-\tilde{G}_{i+1}^{R}\left(x, R_{i+1}(y)\right), R_{i+1}(y)=\left(y_{1}, 2 \zeta_{i+1}-y_{2}\right)^{T}$, has the same singular behavior at $x=y$ as that of the fundamental solution $\tilde{G}_{i+1}^{R}(x, y)$ and decays exponentially at infinite, one can extend the argument in Section 3.1 to show the following inf-sup condition:

$$
\sup _{\psi \in H_{0}^{1}\left(D_{i}^{\mathrm{PML}}\right)} \frac{\left|\left(A_{i-1} \nabla \phi, \nabla \psi\right)-k^{2}\left(J_{i-1} \phi, \psi\right)\right|}{\|\psi\|_{H^{1}\left(D_{i}^{\mathrm{PML}}\right)}} \geq \nu_{i}\|\phi\|_{H^{1}\left(D_{i}^{\mathrm{PML}}\right)}, \quad \forall \phi \in H_{0}^{1}\left(D_{i}^{\mathrm{PML}}\right) .
$$

The inf-sup constant $\nu_{i}$ satisfies $\nu_{i}^{-1} \leq C k^{3 / 2}$. Here we omit the details.

LEMmA 3.8. Let $\sigma_{0} d_{2} \geq 1$ be sufficiently large. We have

$$
\left\|v_{N}-\hat{v}_{N}\right\|_{H^{1}\left(\Omega\left(\zeta_{N},+\infty\right) \cap B_{L}\right)} \leq C k^{\frac{3}{2} N-\frac{1}{2}}(1+k L)^{2} e^{-\frac{1}{2} k \gamma \bar{\sigma}}\|f\|_{H^{1}\left(B_{l}\right)^{\prime}}
$$

Proof. We define

$$
\bar{u}_{N}(x)=-\int_{\Omega_{N}}\left(f_{N}(y)+\bar{f}_{N-1}\right) J_{N-1}(y) G\left(\tilde{x}_{N-1}, \tilde{y}_{N-1}\right) d y .
$$


Then $\bar{u}_{N}=v_{N}$ in $\Omega\left(\zeta_{N},+\infty\right)$ and we have by the similar argument in Lemma 3.6 that

$$
\left\|\bar{u}_{N}\right\|_{H^{1 / 2}\left(\partial \Omega_{N-1}^{\mathrm{PML}}\right)} \leq C k(1+k L) e^{-\frac{1}{2} k \gamma \bar{\sigma}}\|f\|_{H^{1}\left(B_{l}\right)^{\prime}} .
$$

It is easy to see that $\bar{u}_{N}-\hat{v}_{N} \in H^{1}\left(\Omega_{N-1}^{\mathrm{PML}}\right)$ satisfies $\bar{u}_{N}-\hat{v}_{N}=\bar{u}_{N}$ in $\partial \Omega_{N-1}^{\mathrm{PML}}$, and

$$
\left.\left(A_{N-1} \nabla\left(\bar{u}_{N}-\hat{v}_{N}\right)\right), \nabla \psi\right)-k^{2}\left(J_{N-1}\left(\bar{u}_{N}-\hat{v}_{N}\right), \psi\right)=-\left(J_{N-1}\left(\bar{f}_{N-1}-\hat{f}_{N-1}\right), \psi\right),
$$

for any $\psi \in H_{0}^{1}\left(\Omega_{N-1}^{\mathrm{PML}}\right)$. Thus by (3.16) we know that

$$
\begin{aligned}
& \left\|\bar{u}_{N}-\hat{v}_{N}\right\|_{H^{1}\left(\Omega_{N-1}^{\mathrm{PML}}\right)} \\
\leq & C k^{3 / 2}\left\|\bar{f}_{N-1}-\hat{f}_{N-1}\right\|_{H^{-1}\left(\Omega_{N-1}^{\mathrm{PML}}\right)}+C k^{3 / 2}(1+k L)\left\|\bar{u}_{N}\right\|_{H^{1 / 2}\left(\partial \Omega_{N-1}^{\mathrm{PML}}\right)} \\
\leq & C k^{\frac{3}{2} N-\frac{1}{2}}(1+k L)^{2} e^{-\frac{1}{2} k \gamma \bar{\sigma}}\|f\|_{H^{1}\left(B_{l}\right)^{\prime}},
\end{aligned}
$$

where we have used Theorem 3.7 and (3.21) in the last inequality. $\square$

Lemma 3.9. Let $\sigma_{0} d_{2} \geq 1$ be sufficiently large. For $i=3, \cdots, N-1$, we have

$$
\left\|v_{i}-\hat{v}_{i}\right\|_{H^{1}\left(\Omega_{i} \cap B_{L}\right)} \leq C k^{\frac{3}{2}(2 N-i)-\frac{1}{2}}(1+k L)^{2} e^{-\frac{1}{2} k \gamma \bar{\sigma}}\|f\|_{H^{1}\left(B_{l}\right)^{\prime}}
$$

and for $i=2$, we have

$$
\left\|v_{2}-\hat{v}_{2}\right\|_{H^{1}\left(\Omega\left(-\infty, \zeta_{3}\right) \cap B_{L}\right)} \leq C k^{3 N-\frac{7}{2}}(1+k L)^{2} e^{-\frac{1}{2} k \gamma \bar{\sigma}}\|f\|_{H^{1}\left(B_{l}\right)^{\prime}}
$$

Proof. We only prove the case for $i \geq 3$. The case for $i=2$ is similar. We first define the half-space Green function for the PML equation

$$
J_{i-1}^{-1} \nabla \cdot\left(A_{i-1} \nabla \hat{v}_{i}\right)+k^{2} \hat{v}_{i}=f_{i}+\hat{f}_{i-1} \quad \text { in } \Omega\left(-\infty, \zeta_{i+1}\right) .
$$

For $x=\left(x_{1}, x_{2}\right)^{T} \in \mathbb{R}^{2}$, let $\hat{x}_{i+1}^{R}(x)=\left(\hat{x}_{i+1,1}^{R}\left(x_{1}\right), \hat{x}_{i+1,2}^{R}\left(x_{2}\right)\right)^{T}$ be the complex coordinate stretching outside domain $\left(-l_{1}, l_{1}\right) \times\left(\zeta_{i-1}, 2 \zeta_{i+1}-\zeta_{i-1}\right)$, where $\hat{x}_{i+1,1}^{R}\left(x_{1}\right)=$ $\tilde{x}_{1}\left(x_{1}\right)$ and

$$
\hat{x}_{i+1,2}^{R}\left(x_{2}\right)= \begin{cases}x_{2}+\mathbf{i} \int_{\zeta_{i-1}}^{x_{2}} \sigma_{2}\left(t-\zeta_{i-1}+\zeta_{1}\right) d t & \text { if } x_{2}<\zeta_{i-1}, \\ x_{2} & \text { if } \zeta_{i-1} \leq x_{2} \leq 2 \zeta_{i+1}-\zeta_{i-1}, \\ x_{2}+\mathbf{i} \int_{2 \zeta_{i+1}-\zeta_{i-1}}^{x_{2}} \sigma_{2}\left(2 \zeta_{i+1}-\zeta_{i-1}-t+\zeta_{1}\right) d t & \text { if } x_{2}>2 \zeta_{i+1}-\zeta_{i-1}\end{cases}
$$

Denote by $\hat{A}_{i+1}^{R}=\operatorname{diag}\left(\frac{\hat{x}_{i+1,2}^{R}\left(x_{2}\right)^{\prime}}{\hat{x}_{i+1,1}^{R}\left(x_{1}\right)^{\prime}}, \frac{\hat{x}_{i+1,1}^{R}\left(x_{1}\right)^{\prime}}{\hat{x}_{i+1,2}^{R}\left(x_{2}\right)^{\prime}}\right)$ and $\hat{J}_{i+1}^{R}=\hat{x}_{i+1,1}^{R}\left(x_{1}\right)^{\prime} \hat{x}_{i+1,2}^{R}\left(x_{2}\right)^{\prime}$. Obviously $\hat{A}_{i+1}^{R}(x, y)=A_{i}(x, y)$ and $\hat{J}_{i+1}^{R}(x)=J_{i}(x)$ for $x, y \in \Omega\left(-\infty, \zeta_{i+1}\right)$. It is clear that $\hat{G}_{i+1}^{R}(x, y)=\hat{J}_{i+1}^{R}(y) G\left(\hat{x}_{i+1}^{R}(x), \hat{y}_{i+1}^{R}(y)\right)$ is the fundamental solution of the PML equation

$$
\left(\hat{J}_{i+1}^{R}\right)^{-1} \nabla \cdot\left(\hat{A}_{i+1}^{R} \nabla \hat{G}_{i+1}^{R}(x, y)\right)+k^{2} \hat{G}_{i+1}^{R}(x, y)=-\delta_{y}(x) \quad \text { in } \mathbb{R}^{2} .
$$

The Green function of the half-space PML problem is $\hat{\Phi}_{i+1}(x, y)=\hat{G}_{i+1}^{R}(x, y)-$ $\hat{G}_{i+1}^{R}\left(x, R_{i+1}(y)\right)$, where $R_{i+1}(y)=\left(y_{1}, 2 \zeta_{i+1}-y_{2}\right)^{T}$ is the image point of $y$ with respect to $\Gamma_{i+1}$. Define

$$
\begin{aligned}
\bar{v}_{i}(x) & =-\int_{\Omega\left(\zeta_{i-1}, \zeta_{i+1}\right)}\left(f_{i}+\bar{f}_{i-1}\right)(y) \hat{\Phi}_{i+1}(x, y) d y \\
& -\int_{\Gamma_{i+1}} \frac{\partial \hat{\Phi}_{i+1}(x, y)}{\partial y_{2}} v_{i+1}(y) d s(y)=: \bar{v}_{i}^{\mathrm{I}}(x)+\bar{v}_{i}^{\mathrm{II}}(x) \text { in } \Omega\left(-\infty, \zeta_{i+1}\right) .(3.2
\end{aligned}
$$


Then $\bar{v}_{i}=v_{i}$ in $\Omega\left(\zeta_{i-1}, \zeta_{i+1}\right) \cap B_{L}$ by $(2.25)$. By the argument in Lemma 3.6 we can show that

$$
\left\|\bar{v}_{i}^{\mathrm{I}}\right\|_{H^{1 / 2}\left(\partial D_{i}^{\mathrm{PML}} \backslash \Gamma_{i+1}\right)} \leq C k(1+k L) e^{-\frac{1}{2} k \gamma \bar{\sigma}}\|f\|_{H^{1}\left(B_{l}\right)^{\prime}} .
$$

On the other hand, by Theorem 2.8 we have

$$
\begin{aligned}
\bar{v}_{i}^{\mathrm{II}}(x) & =-\int_{\Gamma_{i+1}} \frac{\partial \hat{\Phi}_{i+1}(x, y)}{\partial y_{2}} \tilde{u}(y) d s(y) \\
& =-\int_{\Gamma_{i+1}^{-}} \frac{\partial \hat{\Phi}_{i+1}(x, y)}{\partial y_{2}} \tilde{u}(y) d y_{1}-\int_{\Gamma_{i+1}^{+}} \frac{\partial \hat{\Phi}_{i+1}(x, y)}{\partial y_{2}} \tilde{u}(y) d y_{1},
\end{aligned}
$$

where $\Gamma_{i+1}^{-}=\left\{y=\left(y_{1}, y_{2}\right)^{T} \in \Gamma_{i+1}:\left|y_{1}\right|<l_{1}+d_{2}\right\}$ and $\Gamma_{i+1}^{+}=\left\{y=\left(y_{1}, y_{2}\right)^{T} \in\right.$ $\left.\Gamma_{i+1}:\left|y_{1}\right|>l_{1}+d_{2}\right\}$. By a similar argument in Lemma 3.6 we can show

$$
\left\|\bar{v}_{i}^{\mathrm{II}}\right\|_{H^{1 / 2}\left(\partial D_{i}^{\mathrm{PML}} \backslash \Gamma_{i+1}\right)} \leq C k(1+k L) e^{-\frac{1}{2} k \gamma \bar{\sigma}}\|f\|_{H^{1}\left(B_{l}\right)^{\prime}},
$$

and consequently

$$
\left\|\bar{v}_{i}\right\|_{H^{1 / 2}\left(\partial D_{i}^{\mathrm{PML}} \backslash \Gamma_{i+1}\right)} \leq C k(1+k L) e^{-\frac{1}{2} k \gamma \bar{\sigma}}\|f\|_{H^{1}\left(B_{l}\right)^{\prime}} .
$$

It is clear that $\bar{v}_{i}-\hat{v}_{i} \in H^{1}\left(D_{i}^{\mathrm{PML}}\right)$ satisfies the boundary condition

$$
\bar{v}_{i}-\hat{v}_{i}=\bar{v}_{i+1}-\hat{v}_{i+1} \quad \text { on } \partial D_{i}^{\mathrm{PML}} \cap \Gamma_{i+1}, \quad \bar{v}_{i}-\hat{v}_{i}=\bar{v}_{i} \text { on } \partial D_{i}^{\mathrm{PML}} \backslash \Gamma_{i+1} .
$$

Now we define a lifting function of the boundary value of $\bar{v}_{i}-\hat{v}_{i}$ to the domain $D_{i}^{\mathrm{PML}}$. For $x=\left(x_{1}, x_{2}\right)^{T} \in D_{i}^{\mathrm{PML}}$, define $\phi_{1}(x)=\chi_{i+1}\left(x_{2}\right)\left(\bar{v}_{i+1}-\hat{v}_{i+1}\right)\left(R_{i+1}(x)\right)$, where $\chi_{i+1} \in C_{0}^{\infty}(\mathbb{R})$ is the cut-off function such that $\chi_{i+1}=1$ for $x_{2}>\zeta_{i}, \chi_{i+1}=0$ for $x_{2} \leq \zeta_{i-1}$, and $\left|\chi_{i+1}^{\prime}\right| \leq C \Delta \zeta^{-1}$. Then $\bar{v}_{i}-\hat{v}_{i}-\phi_{1}=0$ on $\partial D_{i}^{\mathrm{PML}} \cap \Gamma_{i+1}$ and $\bar{v}_{i}-\hat{v}_{i}-\phi_{1}=\bar{v}_{i}-\chi_{i+1} \bar{v}_{i+1}$ on $\partial D_{i}^{\mathrm{PML}} \backslash \Gamma_{i+1}$. By (3.2), let $\phi_{2} \in H^{1}\left(D_{i}^{\mathrm{PML}}\right)$ be the lifting function such that $\phi_{2}=\bar{v}_{i}-\chi_{i+1} \bar{v}_{i+1}$ on $\partial D_{i}^{\mathrm{PML}}$ and satisfies $\left\|\phi_{2}\right\|_{H^{1}\left(D_{i}^{\mathrm{PML}}\right)} \leq$ $C\left\|\bar{v}_{i}-\chi_{i+1} \bar{v}_{i+1}\right\|_{H^{1 / 2}\left(\partial D_{i}^{\mathrm{PML}}\right)}$. obtain

By using the inf-sup condition (3.19) and the equation satisfies by $\bar{v}_{i}-\hat{v}_{i}$, we

$$
\begin{aligned}
\left\|\bar{v}_{i}-\hat{v}_{i}\right\|_{H^{1}\left(D_{i}^{\mathrm{PML}}\right)} & \leq \nu_{i}^{-1}\left\|\bar{f}_{i-1}-\hat{f}_{i-1}\right\|_{H^{1}\left(D_{i}^{\mathrm{PML}}\right)}+C\left(1+\nu_{i}^{-1}\right)\left\|\phi_{1}\right\|_{H^{1}\left(D_{i}^{\mathrm{PML}}\right)} \\
& +C\left(1+\nu_{i}^{-1}\right)(1+k L)\left\|\phi_{2}\right\|_{H^{1 / 2}\left(\partial D_{i}^{\mathrm{PML}}\right)} .
\end{aligned}
$$

Since $\bar{v}_{i}-\chi_{i+1} \bar{v}_{i+1}=0$ on $\partial D_{i}^{\mathrm{PML}} \cap \Gamma_{i+1}$, by a similar argument leading to (3.24) we have

$$
\left\|\phi_{2}\right\|_{H^{1 / 2}\left(\partial D_{i}^{\mathrm{PML}}\right)} \leq C k(1+k L) e^{-\frac{1}{2} k \gamma \bar{\sigma}}\|f\|_{H^{1}\left(B_{l}\right)^{\prime}} .
$$

Thus by Theorem 3.7 we finally obtain

$\left\|\bar{v}_{i}-\hat{v}_{i}\right\|_{H^{1}\left(D_{i}^{\mathrm{PML}}\right)} \leq C k^{3 / 2}\left\|\bar{v}_{i+1}-\hat{v}_{i+1}\right\|_{H^{1}\left(D_{i+1}^{\mathrm{PML}}\right)}+C k^{\frac{3}{2} i-\frac{1}{2}}(1+k L)^{2} e^{-\frac{1}{2} k \gamma \bar{\sigma}}\|f\|_{H^{1}\left(B_{l}\right)^{\prime}}$.

This completes the proof by the induction argument and using Lemma 3.8.

The following theorem is a direct consequence of Lemma 3.4, Lemmas 3.8-3.9, and Theorem 2.8. 
Theorem 3.10. Let $\hat{v}=\hat{v}_{N}$ in $\Omega\left(\zeta_{N},+\infty\right) \cap B_{L}$, $\hat{v}=\hat{v}_{i}$ in $\Omega_{i} \cap B_{L}$ for all $i=3, \cdots, N-1$, and $\hat{v}=\hat{v}_{2}$ in $\Omega\left(-\infty, \zeta_{2}\right) \cap B_{L}$. Then for sufficiently large $\sigma_{0} d_{2} \geq 1$, we have

$$
\|\hat{u}-\hat{v}\|_{H^{1}\left(B_{L}\right)} \leq C k^{3 N-\frac{7}{2}}(1+k L)^{2} e^{-\frac{1}{2} k \gamma \bar{\sigma}}\|f\|_{H^{1}\left(B_{l}\right)^{\prime}} .
$$

Let $T$ be the solution operator of (3.3) defined by $T(f)=\hat{u}$ and $\hat{T}$ be the output operator of the STDDM defined by $\hat{T}(f)=\hat{v}$. Then Theorem 3.10 indicates that $\hat{T}$ is a good approximation of $T$ if the PML parameters are chosen such that $k^{3 N-\frac{7}{2}}(1+$ $k L)^{2} e^{-\frac{1}{2} k \gamma \bar{\sigma}}$ is sufficiently small. In this case the discretization of $\hat{T}$ will be a good preconditioner of the corresponding discretization of $T$ if the well-known pollution error [17] of the discretization of the Helmholtz equation is controlled. This idea is confirmed in the numerical examples in the next section.

4. Numerical experiments. In this section we show two numerical examples for both constant and heterogeneous wave number to illustrate the desirable behavior of STDDM as a preconditioner. The computations are all carried out in MATLAB on Dell Precision T5500 with Intel(R) Xeon(R) CPU $2.67 \mathrm{GHz}$ and $72 \mathrm{~GB}$ memory.

Let $l_{1}=l_{2}=0.5, d_{1}=0.2, d_{2}=0.1$ and thus $B_{L}=(-0.7,0.7) \times(-0.6,0.6)$. For the medium property, we set $\bar{l}_{1}=\bar{l}_{2}=0.58$ and define, for $l_{j}<t<\bar{l}_{j}, \sigma_{j}(t)=$ $\hat{\sigma}_{j}(t)+\left(t-l_{j}\right) \hat{\sigma}_{j}^{\prime}(t)$, where

$$
\hat{\sigma}_{j}(t)=\gamma_{0}\left(\int_{l_{j}}^{t}\left(s-l_{j}\right)^{2}\left(\bar{l}_{j}-s\right)^{2} d s\right)\left(\int_{l_{j}}^{\bar{l}_{j}}\left(s-l_{j}\right)^{2}\left(\bar{l}_{j}-s\right)^{2} d s\right)^{-1} .
$$

It is easy to check that $\bar{\sigma}=\gamma_{0} d_{2}$ in (H1).

We use central finite difference scheme to discretize the PML problem (3.3) in $B_{L}$. Let $q$ be mesh density which is the number of nodes put in each wavelength $\lambda=2 \pi / k$. Then the number of nodes in the $x_{j}$ direction is: $n_{j}=q \cdot 2 L_{j} / \lambda, j=1,2$. We set the number of layers $N=10$. The function $\beta_{i}$ in $\Omega_{i}$ in the definition of the source transfer algorithm is discretized by setting $\beta_{i}=\beta_{i}^{h}$ at the nodes, where $\beta_{i}^{h}=\beta_{i}^{h}\left(x_{2}\right)$ is a piecewise linear function which equals to 1 in the first one fourth rows near $\Gamma_{i}, 0$ in the last one fourth rows near $\Gamma_{i+1}$, and linear in the middle rows. It is clear that $\left|\left(\beta_{i}^{h}\right)^{\prime}\right| \leq C(\Delta \zeta)^{-1}$. We remark that this choice of the profile for $\beta_{i}$ is convenient for our computation since given $\beta_{i}^{h}$, one can easily construct a smooth function $\beta_{i}$ such that $\left|\beta_{i}^{\prime}\right| \leq C(\Delta \zeta)^{-1}$ with the constant $C$ independent of the mesh size and $\beta_{i}=\beta_{i}^{h}$ at the nodes.

We use our STDDM as the preconditioner of the GMRES method for solving the discretized problem of (3.3). The relative residue tolerance in the GMRES solver is set to be $10^{-10}$. The linear system of equations in each subdomain is solved by MUMPS [1, 2].

Example 4.1. In this example we test the algorithm for the problem with constant wave number $k / 2 \pi=50,100$. We take $\gamma_{0}$ such that the exponential decaying factor $k^{3 N-7 / 2}(1+k L)^{2} e^{-\frac{1}{2} k \gamma \gamma_{0} d_{2}} \leq 10^{-2}$ to guarantee the effectiveness of STDDM by Theorem 3.10 .

We set the external force $f(x)$ to be a narrow Gaussian point source located at $\left(r_{1}, r_{2}\right)=(0.2,0.1)$ :

$$
f\left(x_{1}, x_{2}\right)=e^{-\left(\frac{4 k}{\pi}\right)^{2}\left(\left(x_{1}-r_{1}\right)^{2}+\left(x_{2}-r_{2}\right)^{2}\right)} .
$$




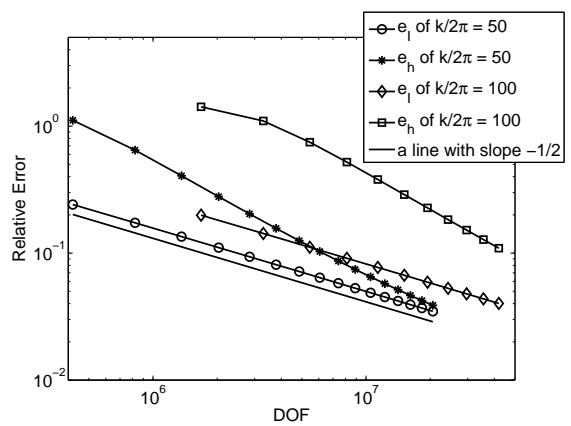

(a)

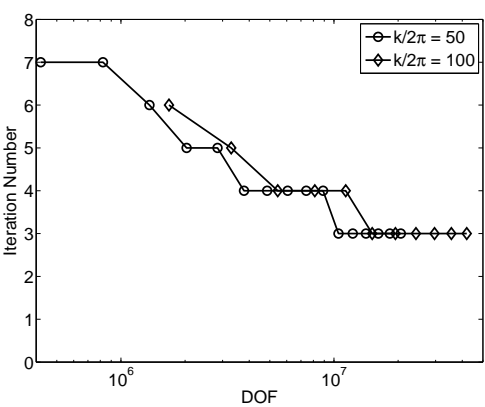

(b)

Fig. 4.1. (a) The relative error $e_{I}$ and $e_{h}$ in terms of the number of degree freedom $\mathrm{DOF}=$ $n_{1} n_{2}$; (b) The number of iterations of the preconditioned GMRES algorithm using STDDM as the preconditioner. The mesh density $q=10,14, \ldots, 70$ when $k / 2 \pi=50$ and $q=10,14, \ldots, 50$ when $k / 2 \pi=100$.

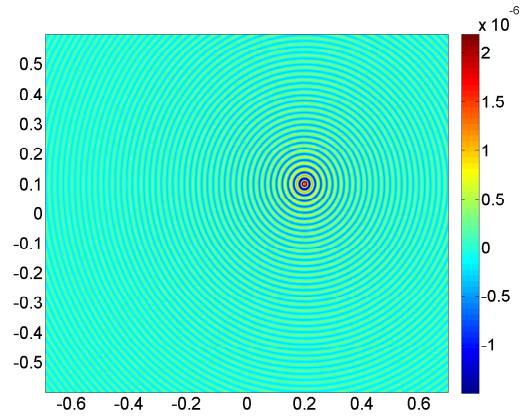

(a)

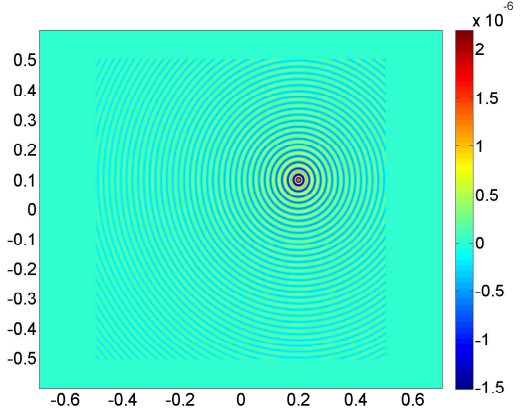

(b)

FIG. 4.2. (a) The real part of the interpolation solution; (b) The real part of the numerical solution.

We denote by $u$ the exact solution and $u_{I}$ the linear interpolation of $u$ on a triangular mesh over $B_{l}$ which is obtained by connecting the lower-left node and the upperright node of the finite difference grid cells. Let $u_{h}$ be the discrete solution which is piecewise linear on the mesh with nodal values equal to the finite difference solution. We compute the relative error of the interpolation solution and the discrete solution:

$$
e_{I}=\left|u-u_{I}\right|_{H^{1}\left(B_{l}\right)} /|u|_{H^{1}\left(B_{l}\right)}, \quad e_{h}=\left|u-u_{h}\right|_{H^{1}\left(B_{l}\right)} /|u|_{H^{1}\left(B_{l}\right)} .
$$

Here the integrals are computed by using a third order quadrature formula on each element of the mesh. The values of $\nabla u$ at the quadrature points are computed using the integral representation formula with the source $f$.

Figure 4.1(a) shows the error decay of the interpolation solution and the discrete solution when the mesh density $q$ increases. Here the number of the degrees of freedom $\mathrm{DOF}=n_{1} n_{2}$. Figure 4.1(b) shows the number of preconditioned GMRES iterations in terms of DOF. They indicate clearly that the number of iterations decreases when the pollution error decreases. In Figure 4.2 we show the real part of the interpolation solution and the discrete solution when $k / 2 \pi=50$ and $q=50$.

Example 4.2. We consider in this example the Helmholtz equation with het- 


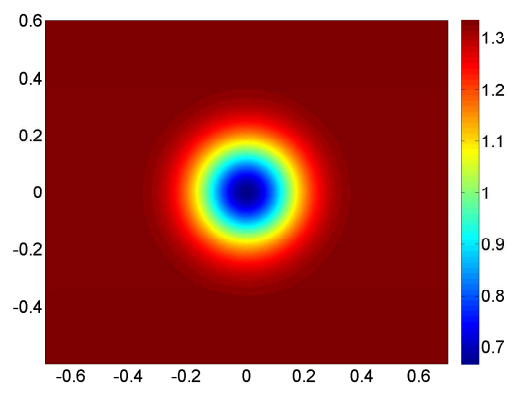

(a)

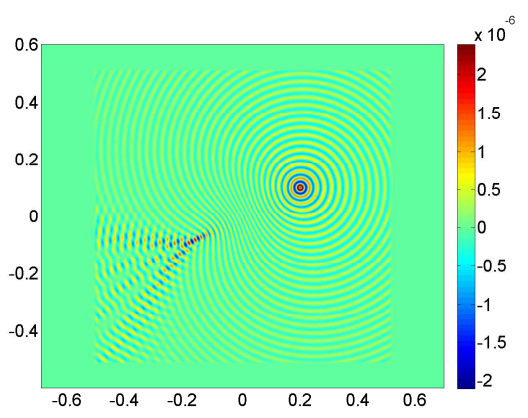

(b)

FIG. 4.3. (a) The velocity field; (b) The real part of the numerical solution observed from the direction $[0,0,1]$.

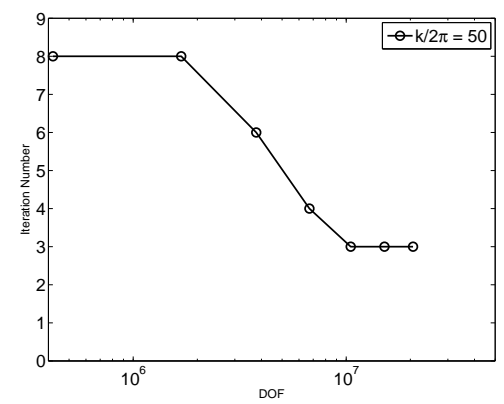

FIG. 4.4. The number of iterations of the preconditioned GMRES algorithm using STDDM as the preconditioner for heterogeneous wave number. The mesh density $q=10,20, \ldots, 70$.

erogeneous wave number $k(x)=\omega / c(x)$, where $\omega$ is the angular frequency and $c(x)$ is the velocity field. We set the external force $f(x)$ to be a narrow Gaussian point source located at $\left(r_{1}, r_{2}\right)=(0.2,0.1)$ :

$$
f\left(x_{1}, x_{2}\right)=e^{-\left(\frac{4 \omega}{\pi}\right)^{2}\left(\left(x_{1}-r_{1}\right)^{2}+\left(x_{2}-r_{2}\right)^{2}\right)} .
$$

We take $\gamma_{0}$ so that the decaying factor $\omega^{3 N-7 / 2}(1+\omega L)^{2} e^{-\frac{1}{2} \omega \gamma \gamma_{0} d_{2}} \leq 10^{-2}$. We consider $\omega / 2 \pi=50$ and the velocity field that used in [14]: A smooth converging lens with a Gaussian profile at the center $\left(r_{1}, r_{2}\right)=(0,0)$ (see Figure 4.3 (a)):

$$
c(x)=\frac{4}{3}-\frac{2}{3} e^{-20\left(\left(x_{1}-r_{1}\right)^{2}+\left(x_{2}-r_{2}\right)^{2}\right)} .
$$

Since $2 / 3 \leq c(x) \leq 4 / 3$, we set $\lambda=2 \pi / \omega$ as the average wavelength and $n_{j}=$ $q \cdot 2 L_{j} / \lambda$. Figure $4.3(\mathrm{~b})$ shows the real part of numerical solution when $q=50$. The number of iterations of the preconditioned GMRES algorithm using STDDM as the preconditioner is shown in Figure 4.4 which again shows the decrease of the iteration numbers with the increase of the discretization accuracy.

5. Appendix: The proof of Lemma 2.6. We start by proving the following elementary lemma. 
Lemma 5.1. For $j=1,2$, if either $\left|x_{j}\right| \geq M$ or $\left|y_{j}\right| \geq M$, we have

$$
\left|\int_{y_{j}}^{x_{j}} \sigma_{j}(t) d t\right| \geq \gamma_{0}\left|x_{j}-y_{j}\right|-2\left(\sigma_{0}+\gamma_{0}\right) M .
$$

Proof. We only prove the case $j=1$. Without loss of generality we may assume $\left|x_{1}\right| \geq M$. Now if $\left|y_{1}\right| \geq M$, we know that

$$
\left|\int_{y_{1}}^{x_{1}} \sigma_{1}(t) d t\right| \geq \begin{cases}\gamma_{0}\left|x_{1}-y_{1}\right| & \text { if } x_{1} y_{1}>0 \\ \gamma_{0}\left|x_{1}-y_{1}\right|-2\left(\sigma_{0}+\gamma_{0}\right) M & \text { if } x_{1} y_{1}<0\end{cases}
$$

On the other hand, if $\left|y_{1}\right| \leq M$, we have

$$
\begin{aligned}
\left|\int_{y_{1}}^{x_{1}} \sigma_{1}(t) d t\right| & \geq\left|\int_{M}^{\left|x_{1}\right|} \sigma_{1}(t) d t\right|-\left|\int_{-\left|y_{1}\right|}^{M} \sigma_{1}(t) d t\right| \\
& \geq \gamma_{0}\left(\left|x_{1}\right|-M\right)-\sigma_{0}\left(M+\left|y_{1}\right|\right) \\
& \geq \gamma_{0}\left|x_{1}-y_{1}\right|-2\left(\gamma_{0}+\sigma_{0}\right) M .
\end{aligned}
$$

This completes the proof. $\square$

Lemma 5.2. For $i=1, \cdots, N-1$, we have

$$
\operatorname{Im} \rho(\tilde{x}, \tilde{y}) \geq \frac{1}{2} \gamma_{0}|x-y|-\sqrt{2}\left(\sigma_{0}+\gamma_{0}\right) M, \quad \forall|x| \geq M_{i},|y| \leq M_{i-1}
$$

Proof. If $\left|x_{1}-y_{1}\right| \geq\left|x_{2}-y_{2}\right|$, we have $\left|x_{1}-y_{1}\right| \geq|x-y| / \sqrt{2}$ and

$$
\left|x_{1}\right| \geq \frac{1}{\sqrt{2}}|x-y|-\left|y_{1}\right| \geq \frac{1}{\sqrt{2}}\left(M_{i}-M_{i-1}\right)-M_{i-1} \geq M .
$$

The lemma follows from (2.5) and Lemma 5.1

$$
\operatorname{Im} \rho(\tilde{x}, \tilde{y}) \geq \frac{1}{\sqrt{2}}\left|\int_{y_{1}}^{x_{1}} \sigma_{1}(t) d t\right| \geq \frac{1}{2} \gamma_{0}|x-y|-\sqrt{2}\left(\sigma_{0}+\gamma_{0}\right) M .
$$

This completes the proof. $\square$

We are now in the position to complete the proof of Lemma 2.6.

Proof of Lemma 2.6. We will use the induction argument. First we consider the case when $i=1$. Since $f_{1}$ is supported in $B_{l}$, by Lemma 2.5 and the fact that $|x-y| \geq \sqrt{2}(M+l)$ for $|x| \geq M_{1}$ and $y \in B_{l}$ we have

$$
\begin{aligned}
& \left|u_{1}(x)\right|+\left|\nabla u_{1}(x)\right| \\
\leq & C\left\|f_{1}\right\|_{H^{1}\left(B_{l}\right)^{\prime}} \max _{y \in B_{l}}\left(|G(\tilde{x}, \tilde{y})|+\left|\nabla_{y} G(\tilde{x}, \tilde{y})\right|+\left|\nabla_{x} G(\tilde{x}, \tilde{y})\right|+\left|\nabla_{x} \nabla_{y} G(\tilde{x}, \tilde{y})\right|\right) \\
\leq & C e^{-\frac{1}{2} k \operatorname{Im} \rho(\tilde{x}, \tilde{y})}\left\|f_{1}\right\|_{H^{1}\left(B_{l}\right)^{\prime}}
\end{aligned}
$$

This implies by Lemma 5.2 that for any $|x| \geq M_{1}$,

$$
\left|u_{1}(x)\right|+\left|\nabla u_{1}(x)\right| \leq C e^{-\frac{1}{4} k \gamma_{0}|x|}\left\|f_{1}\right\|_{H^{1}\left(B_{l}\right)^{\prime}} \leq C e^{-\frac{1}{8} k \gamma_{0}|x|}\|f\|_{H^{1}\left(B_{l}\right)^{\prime}} .
$$

The estimate $\left\|u_{1}\right\|_{H^{1}\left(\mathbb{R}^{2}\right)} \leq C\|f\|_{H^{1}\left(B_{l}\right)^{\prime}}$ follows from the above estimate and the definition of $u_{1}$. 
Now we show the estimate for $u_{i}, i \geq 2$. We only prove the estimate for $u_{i}$. The estimate for $\nabla u_{i}$ is similar. From the definition of $u_{i}$ and (2.17) we know that

$$
\left|u_{i}(x)\right| \leq\left|\int_{\Omega_{i}} f_{i}(y) \tilde{G}(x, y) d y\right|+C \int_{\Omega_{i}}\left(\left|u_{i-1}(y)\right|+\left|\nabla u_{i-1}(y)\right|\right)|\tilde{G}(x, y)| d y:=\mathrm{I}+\mathrm{II} .
$$

Since $f_{i}$ is supported in $B_{l}$, by the same argument in deriving (5.2), we have

$$
\mathrm{I} \leq C e^{-\frac{1}{4} k \gamma_{0}|x|}\|f\|_{H^{1}\left(B_{l}\right)^{\prime}} .
$$

To estimate II we first split the integral into two parts, where $B\left(M_{i-1}\right)$ is the circle of radius $M_{i-1}$,

$$
\begin{aligned}
\mathrm{II} & =C \int_{\Omega_{i} \cap B\left(M_{i-1}\right)}\left(\left|u_{i-1}(y)\right|+\left|\nabla u_{i-1}(y)\right|\right)|\tilde{G}(x, y)| d y \\
& +C \int_{\Omega_{i} \backslash \bar{B}\left(M_{i-1}\right)}\left(\left|u_{i-1}(y)\right|+\left|\nabla u_{i-1}(y)\right|\right)|\tilde{G}(x, y)| d y:=\mathrm{II}_{1}+\mathrm{II}_{2} .
\end{aligned}
$$

By the induction assumption $\left\|u_{i-1}\right\|_{H^{1}\left(\mathbb{R}^{2}\right)} \leq C\|f\|_{H^{1}\left(B_{l}\right)^{\prime}}$ we know that

$$
\mathrm{II}_{1} \leq C\left\|u_{i-1}\right\|_{H^{1}\left(\mathbb{R}^{2}\right)} \max _{|y| \leq M_{i-1}}|\tilde{G}(x, y)| \leq C e^{-\frac{1}{2} k \operatorname{Im} \rho(\tilde{x}, \tilde{y})}\|f\|_{H^{1}\left(B_{l}\right)^{\prime}}
$$

where we have used (2.6) and the fact that $|x-y| \geq \sqrt{2}\left(M+M_{i-1}\right)$ for $|x| \geq M_{i}$ and $|y| \leq M_{i-1}$. Now Lemma 5.2 implies

$$
\mathrm{II}_{1} \leq C e^{-\frac{1}{4} k \gamma_{0}|x|}\|f\|_{H^{1}\left(B_{l}\right)^{\prime}} .
$$

Next by the induction assumption

$$
\left|u_{i-1}(y)\right|+\left|\nabla u_{i-1}(y)\right| \leq C e^{-\frac{1}{8} k \gamma_{0}|y|}\|f\|_{H^{1}\left(B_{l}\right)^{\prime}} \quad \text { for }|y| \geq M_{i-1},
$$

and (2.6), we have

$$
\mathrm{II}_{2} \leq C\|f\|_{H^{1}\left(B_{l}\right)^{\prime}} \int_{\Omega_{i} \backslash \bar{B}\left(M_{i-1}\right)} e^{-\frac{1}{8} k \gamma_{0}|y|-\frac{1}{2} k \operatorname{Im} \rho(\tilde{x}, \tilde{y})} \frac{1}{|x-y|^{1 / 2}} d y .
$$

If $\left|x_{1}-y_{1}\right| \geq\left|x_{2}-y_{2}\right|$, for $y \in \Omega_{i} \backslash \bar{B}\left(M_{i-1}\right)$, since $\left|y_{2}\right| \leq l$, we have $\left|y_{1}\right| \geq M$, by Lemma 5.1

$$
\operatorname{Im} \rho(\tilde{x}, \tilde{y}) \geq \frac{1}{\sqrt{2}}\left|\int_{y_{1}}^{x_{1}} \sigma_{1}(t) d t\right| \geq \frac{1}{2} \gamma_{0}|x-y|-\sqrt{2}\left(\sigma_{0}+\gamma_{0}\right) M,
$$

and consequently

$$
\begin{aligned}
\mathrm{II}_{2} & \leq C\|f\|_{H^{1}\left(B_{l}\right)^{\prime}} \int_{\Omega_{i} \backslash \bar{B}\left(M_{i-1}\right)} e^{-\frac{1}{8} k \gamma_{0}|y|-\frac{1}{4} k \gamma_{0}|x-y|} \frac{1}{|x-y|^{1 / 2}} d y \\
& \leq C\|f\|_{H^{1}\left(B_{l}\right)^{\prime}} e^{-\frac{1}{8} k \gamma_{0}|x|} \int_{\Omega_{i} \backslash \bar{B}\left(M_{i-1}\right)} e^{-\frac{1}{8} k \gamma_{0}|x-y|} \frac{1}{|x-y|^{1 / 2}} d y \\
& \leq C e^{-\frac{1}{8} k \gamma_{0}|x|}\|f\|_{H^{1}\left(B_{l}\right)^{\prime}},
\end{aligned}
$$

where we have used the triangle inequality $|x-y|+|y| \geq|x|$. 
If $\left|x_{2}-y_{2}\right| \geq\left|x_{1}-y_{1}\right|$ and $\left|x_{2}\right| \geq M$, we again by Lemma 5.1 have

$$
\operatorname{Im} \rho(\tilde{x}, \tilde{y}) \geq \frac{1}{\sqrt{2}}\left|\int_{y_{2}}^{x_{2}} \sigma_{2}(t) d t\right| \geq \frac{1}{2} \gamma_{0}|x-y|-\sqrt{2}\left(\sigma_{0}+\gamma_{0}\right) M
$$

which implies by the similar argument in (5.3) that

$$
\mathrm{II}_{2} \leq C e^{-\frac{1}{8} k \gamma_{0}|x|}\|f\|_{H^{1}\left(B_{l}\right)^{\prime}} .
$$

Finally, if $\left|x_{2}-y_{2}\right| \geq\left|x_{1}-y_{1}\right|$ and $\left|x_{2}\right| \leq M$, since $\left|y_{2}\right| \leq l$ for $y \in \Omega_{i} \backslash \bar{B}\left(M_{i-1}\right)$, we have $|x-y| \leq \sqrt{2}(M+l)$. Thus

$$
\begin{aligned}
\mathrm{II}_{2} & \leq C\|f\|_{H^{1}\left(B_{l}\right)^{\prime}} \int_{B(x, \sqrt{2}(M+l))} e^{-\frac{1}{8} k \gamma_{0}|y|} \frac{1}{|x-y|^{1 / 2}} d y \\
& \leq C e^{-\frac{1}{8} k \gamma_{0}|x|}\|f\|_{H^{1}\left(B_{l}\right)^{\prime}} \int_{B(x, \sqrt{2}(M+l))} \frac{1}{|x-y|^{1 / 2}} d y \\
& \leq C e^{-\frac{1}{8} k \gamma_{0}|x|}\|f\|_{H^{1}\left(B_{l}\right)^{\prime}},
\end{aligned}
$$

where we have used $|y| \geq|x|-|x-y| \geq|x|-\sqrt{2}(M+l)$ for $y \in B(x, \sqrt{2}(M+l))$. This completes the proof.

Acknowledgement. The authors would like to thank the referees for their insightful comments that greatly improved the paper.

\section{REFERENCES}

[1] P. R. Amestoy, I.S. Duff, J. Koster and J.-Y. L'Excellent, A fully asynchronous multifrontal solver using distributed dynamic scheduling, SIAM J. Matrix Anal. Appl., 2001, 15-41.

[2] P. R. Amestoy, A. Guermouche, J.-Y. L'Excellent and S. Pralet, Hybrid scheduling for the parallel solution of linear systems, Parallel Computing, 2006, 136-156.

[3] J.H. Bramble and J.E. Pasciak, Analysis of a Cartesian PML approximation to acoustic scattering problems in $\mathbb{R}^{2}$ and $\mathbb{R}^{3}$, J. Appl. Comput. Math. 247 (2013), 209-230.

[4] J.-D. Benamou and B. Després, A domain decomposition method for the Helmholtz equation and related optimal control problems, J. Comput. Phys. 136 (1997), 68-82.

[5] J. P. Bérenger, A perfectly matched layer for the absorption of electromagnetic waves, J. Comput. Phys. 114 (1994), 185-200.

[6] A. Brandt and I. Livshits, Wave-ray multigrid method for standing wave equations, Electronic Trans. Numer. Anal. 6 (1997), 162-181.

[7] S.N. Chandler-Wilde, I.G. Graham, S. Langdon, and M. Lindner, Condition number estimates for combined potential boundary integral operators in acoustic scattering, J. Integral Equa. Appli. 21 (2009), 229-279.

[8] W.C. Chew and W. Weedon, A 3D perfectly matched medium from modified Maxwell's equations with stretched coordinates. Microwave Opt. Tech. Lett. 7 (1994), 599-604.

[9] Z. Chen and X. Liu, An adaptive perfectly matched layer technique for time-harmonic scattering problems, SIAM J. Numer. Anal. 41 (2003), 799-826.

[10] Z. Chen and X.M. Wu, An adaptive uniaxial perfectly matched layer technique for TimeHarmonic Scattering Problems, Numerical Mathematics: Theory, Methods and Applications, 1 (2008), 113-137.

[11] Z. Chen and X. Xiang, A source transfer domain decomposition method for Helmholtz equations in unbounded domain, Part II: Extensions, Numerical Mathematics: Theory, Methods and Applications, to appear.

[12] Z. Chen and W. Zheng, Convergence of the uniaxial perfectly matched layer method for timeharmonic scattering problems in two-layered media, SIAM J. Numer. Anal, 48 (2011), 21582185 .

[13] H.C. Elman, O.G. Ernst, and D.P. O'Leary, A multigrid nethod enhanced by Krylov subspace iteration for discrete Helmholtz equations, SIAM J. Sci. Comput. 23 (2001), 1291-1315.

[14] B. Engquist and L. Ying, Sweeping preconditioner for the Helmholtz equation: Moving perfectly matched layers, Multiscle Model. Simul. 9 (2011), 686-710. 
[15] Y. A. Erlangga, Advances in iterative methods and preconditioners for the Helmholtz equation, Arch. Comput. Methods Eng. 15 (2008), 37-66.

[16] M.J. Gander, F. Magoules, and F. Nataf, Optimized Schwarz methods without overlap for the Helmholtz equation, SIAM J.Sci. Comput. (2002), 38-60.

[17] F. Ihlenburg, Finite Element Analysis of Acoustic Scattering, Springer, New York, 1988.

[18] S. Kim and J.E. Pasciak, Analysis of a Cartisian PML approximation to acoustic scattering problems in $\mathbb{R}^{2}$, J. Math. Anal. Appl. 370 (2010), 168-186.

[19] M. Lassas and E. Somersalo, On the existence and convergence of the solution of PML equations. Computing 60 (1998), 229-241.

[20] N.N. Lebedev, Special Fuctions and Their Applications, Dover Publishing, New York, 1972.

[21] M. Lassas and E. Somersalo, Analysis of the PML equations in general convex geometry. Proc. Roy. Soc. Eding. 131 (2001), 1183-1207.

[22] D. Osei-Kuffuor and Y. Saad, Preconditioning Helmholtz linear systems, Technical Report, umsi-2009-30, Minnesota Supercomputer Institute, University of Minnesota, 2009.

[23] F.L. Teixeira and W.C. Chew, Advances in the theory of perfectly matched layers, Fast and Efficient Algorithms in Computational Electromagnetics, 2001, 283-346.

[24] G.N. Watson, A Treatise on the Theory of Bessel Functions, Cambridge University Press, Cambridge, 1922. 